\title{
Allylic Alkylation and Ring-Closing Metathesis in Sequence: a Successful Cohabitation of Pd and Ru
}

Claire Kammerer, Guillaume Prestat*, Thomas Gaillard, David Madec and Giovanni Poli*

Laboratoire de Chimie Organique UMR CNRS 7611

Institut de Chimie Moléculaire FR2769

Université Pierre et Marie Curie-Paris 6, Case 183

4, Place Jussieu, F-75252 Paris, France

guillaume.prestat@upmc.fr, giovanni.poli@upmc.fr

\section{Supporting Information}




\section{Table of contents}

I. General remarks $\quad$ S3

II. Preparation of starting materials $\quad$ S3

$\begin{array}{ll}\text { Dimethyl 2-allylmalonate 1a } & \text { S3 }\end{array}$

Dimethyl 2-(but-3'-enyl)malonate 1b $\quad$ S4

Dimethyl 2-(pent-4'-enyl)malonate 1c $\quad$ S4

III. Typical procedures $\quad$ S5

III.1. Typical procedures for the pseudo domino reactions $\quad$ S5

"Allylic alkylation - ring-closing metathesis" PDOM sequence $\quad$ S5

"Allylic alkylation - allylic alkylation - ring-closing metathesis" PDOM sequence S5

III.2. Typical procedure for the allylic alkylation with ruthenium $\quad$ S6

IV. Characterization data for the reaction products S6

Dimethyl 2-allylmalonate 1a $\quad$ S6

$\begin{array}{ll}\text { Dimethyl cyclopent-3-ene-1,1-dicarboxylate 2a } & \text { S7 }\end{array}$

$\begin{array}{ll}\text { Dimethyl cyclohex-3-ene-1,1-dicarboxylate } \mathbf{2 b} & \text { S7 }\end{array}$

$\begin{array}{ll}\text { Dimethyl cyclohept-3-ene-1,1-dicarboxylate 2c } & \text { S7 }\end{array}$

$\begin{array}{ll}\text { Dimethyl 2,2-diallylmalonate } 3 & \text { S8 }\end{array}$

1,1-diacetylcyclopent-3-ene $\mathbf{1 3} \quad$ S8

$\begin{array}{ll}\text { Methyl 1-acetyl-cyclopent-3-enecarboxylate } 14 & \text { S8 }\end{array}$

$\begin{array}{ll}\text { Ethyl-1-nitro-cyclopent-3-enecarboxylate } \mathbf{1 5} & \text { S9 }\end{array}$

8,8-dimethyl-7,9-dioxaspiro[4.5]dec-2-ene-6,10-dione $16 \quad$ S9

Spiro[4.5]dec-2-ene-6,10-dione $17 \quad$ S9

7,9-dimethyl-9-diazaspiro[4.5]dec-2-ene-6,8,10-trione $\mathbf{1 8}$ S10

V. ${ }^{1} \mathrm{H}$ and ${ }^{13} \mathrm{C}$ NMR spectra $\quad \mathbf{S 1 0}$ 


\section{General remarks}

Dichloromethane was distilled on $\mathrm{CaH}_{2}$. THF and toluene were distilled on sodium/benzophenone. Tetrakis(triphenylphosphine)palladium was synthesized according to a published procedure. ${ }^{1}$ All other reagents and solvents were used without further purification. ${ }^{1} \mathrm{H}$ NMR $(400 \mathrm{MHz})$ and ${ }^{13} \mathrm{C} \mathrm{NMR}(100 \mathrm{MHz})$ were recorded on a Brüker ARX-400 spectrometer using the residual peak of chloroform- $d$ as internal standard (7.27 ppm for ${ }^{1} \mathrm{H}$ NMR and $77.2 \mathrm{ppm}$ for ${ }^{13} \mathrm{C}$ NMR). Chemical shifts are reported in ppm and coupling constants $J$ in Hertz. IR spectra were recorded on a Brüker Tensor 27 (pike) instrument and only the strongest or structurally most important peaks were listed. Flash chromatography was conducted using 40-63 $\mu \mathrm{m}$ silica. Analytical TLC were performed on Merck precoated silica $60-\mathrm{F}_{254}$ plates.

\section{Preparation of starting materials}

\section{Dimethyl 2-allylmalonate 1a}

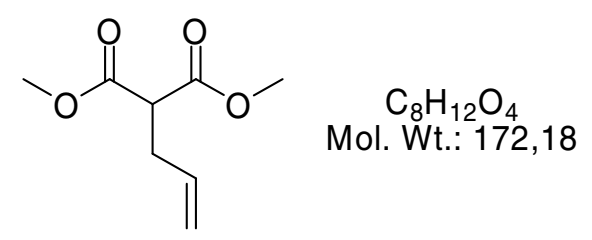

Dimethyl malonate ( $1 \mathrm{~mL}, 8.8 \mathrm{mmol}, 1.5$ equiv.) and 3-bromoprop-1-ene (0.5 mL, $5.8 \mathrm{mmol}, 1$ equiv.) were added to a solution of potassium carbonate $(2.4 \mathrm{~g}, 17.4 \mathrm{mmol}, 3$ equiv.) in acetone $(30 \mathrm{~mL})$. The reaction mixture was allowed to stir for $24 \mathrm{~h}$ at room temperature and was then quenched with a saturated solution of $\mathrm{NH}_{4} \mathrm{Cl}$. The organic layer was separated and the aqueous layer extracted with methylene chloride. The combined organic layers were washed with brine, dried over $\mathrm{MgSO}_{4}$ and concentrated under reduced pressure. The crude product was purified by flash chromatography on silica gel eluting with cyclohexane / AcOEt $80: 20$ to afford $917 \mathrm{mg}(91 \%)$ of the expected product 1a as a yellow oil.

Data in agreement with: Curran, D. P.; Fairweather, N. J. Org. Chem. 2003, 68, 2972-2974. Commercially available.

${ }^{1} \mathbf{H}$ NMR $\left(\mathbf{C D C l}_{3}, 400 \mathrm{MHz}\right): \delta=2.55(\mathrm{tt}, J=1.5,7.5 \mathrm{~Hz}, 2 \mathrm{H}), 3.38(\mathrm{t}, J=7.5 \mathrm{~Hz}, 1 \mathrm{H}), 3.64(\mathrm{~s}, 6 \mathrm{H}), 4.96(\mathrm{dd}$, $J=1.0,10.5 \mathrm{~Hz}, 1 \mathrm{H}), 5.02(\mathrm{ddt}, J=1.5,2.0,17.0 \mathrm{~Hz}, 1 \mathrm{H}), 5.68(\mathrm{ddt}, J=7.0,10.0,17.0 \mathrm{~Hz}, 1 \mathrm{H})$.

${ }^{13} \mathbf{C}$ NMR $\left(\mathbf{C D C l}_{3}, 100 \mathrm{MHz}\right): \delta=32.8,51.2,52.3,117.5,133.9,169.1$.

IR (ATR) : 2955, 1732, 1644, $1435 \mathrm{~cm}^{-1}$

\footnotetext{
${ }^{1}$ Coulson, D. R. Inorg. Synth. 1972, 13, 121-124.
} 


\section{Dimethyl 2-(but-3'-enyl)malonate 1b}

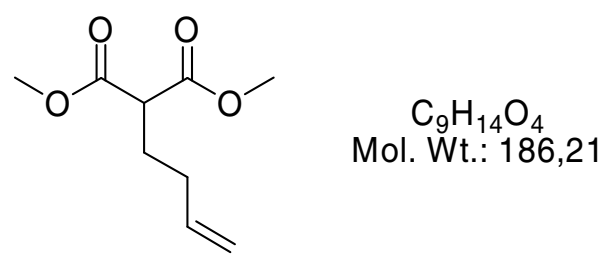

At $0{ }^{\circ} \mathrm{C}$, dimethyl malonate $(1 \mathrm{~mL}, 8.8 \mathrm{mmol}, 1$ equiv.) was added dropwise to a suspension of sodium hydride (390 mg, 9.7 mmol, 1.1 equiv., 60\% in mineral oil) in DMF (20 mL). The mixture was allowed to warm up to room temperature and 4-bromobut-1-ene $(1.05 \mathrm{~mL}, 8.8 \mathrm{mmol}, 1$ equiv.) was introduced. The reaction mixture was allowed to stir for $24 \mathrm{~h}$ at room temperature and was then quenched with a saturated solution of $\mathrm{NH}_{4} \mathrm{Cl}$. The organic layer was separated and the aqueous layer extracted with diethyl ether. The combined organic layers were washed with brine, dried over $\mathrm{MgSO}_{4}$ and concentrated under reduced pressure. The crude product was purified by flash chromatography on silica gel eluting with pentane $/ \mathrm{Et}_{2} \mathrm{O} 95: 5$ to afford $1.14 \mathrm{~g}$ $(70 \%)$ of the expected product $\mathbf{1 b}$ as a yellow oil.

Data in agreement with: Laidig, G. J.; Hegedus, L. S. Synthesis 1995, 527-532.

${ }^{1} \mathbf{H}$ NMR $\left(\mathbf{C D C l}_{3}, 400 \mathrm{MHz}\right): \delta=1.99-2.13(\mathrm{~m}, 4 \mathrm{H}), 3.41(\mathrm{t}, J=7.5 \mathrm{~Hz}, 1 \mathrm{H}), 3.75(\mathrm{~s}, 6 \mathrm{H}), 5.00-5.07(\mathrm{~m}$, 2H), 5.77 (ddt, $J=6.5,10.5,16.5 \mathrm{~Hz}, 1 \mathrm{H})$.

${ }^{13} \mathbf{C ~ N M R}\left(\mathbf{C D C l}_{3}, \mathbf{1 0 0} \mathbf{M H z}\right): \delta=28.0,31.4,51.0,52.6,116.2,136.9,170.0$.

IR (ATR) : 2955, 1732, 1642, $1435 \mathrm{~cm}^{-1}$

\section{Dimethyl 2-(pent-4'-enyl)malonate 1c}

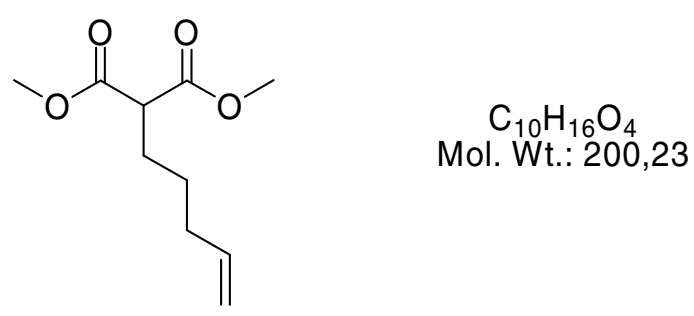

At $0{ }^{\circ} \mathrm{C}$, dimethyl malonate $(0.5 \mathrm{~mL}, 4.4 \mathrm{mmol}, 1$ equiv.) was added dropwise to a suspension of sodium hydride (196 mg, $4.9 \mathrm{mmol}, 1.1$ equiv., 60\% in mineral oil) in DMF (10 mL). The mixture was allowed to warm up to room temperature and 5-bromopent-1-ene $(0.4 \mathrm{~mL}, 3.4 \mathrm{mmol}, 0.8$ equiv. $)$ was introduced. The reaction mixture was allowed to stir for $24 \mathrm{~h}$ at room temperature and was then quenched with a saturated solution of $\mathrm{NH}_{4} \mathrm{Cl}$. The organic layer was separated and the aqueous layer extracted with diethyl ether. The combined organic layers were washed with brine, dried over $\mathrm{MgSO}_{4}$ and concentrated under reduced pressure. The crude product was purified by flash chromatography on silica gel eluting with pentane / $\mathrm{Et}_{2} \mathrm{O} 95: 5$ to afford $292 \mathrm{mg}(43 \%)$ of the expected product 1c as a yellow oil. 
Data in agreement with: Fournet, G.; Balme, G.; Gore, J. Tetrahedron 1990, 46, 7763-7774.

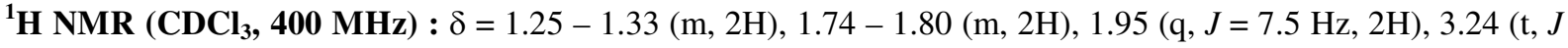
$=7.5 \mathrm{~Hz}, 1 \mathrm{H}), 3.59(\mathrm{~s}, 6 \mathrm{H}), 4.82(\mathrm{br} \mathrm{d}, J=10.0 \mathrm{~Hz}, 1 \mathrm{H}), 4.88(\mathrm{dd}, J=1.5,17.0 \mathrm{~Hz}, 1 \mathrm{H}), 5.64(\mathrm{ddt}, J=6.5,10.0$, $17.0 \mathrm{~Hz}, 1 \mathrm{H})$.

${ }^{13} \mathbf{C ~ N M R}\left(\mathbf{C D C l}_{3}, \mathbf{1 0 0} \mathbf{M H z}\right): \delta=26.3,28.1,33.0,51.3,52.1,114.8,137.6,169.5$.

IR (ATR) : 2954, 1734, 1641, $1435 \mathrm{~cm}^{-1}$

\section{Typical procedures}

\section{III.1. Typical procedures for the pseudo domino reactions}

\section{"Allylic alkylation - ring-closing metathesis" PDOM sequence}

The palladium catalyst was dissolved in $0.5 \mathrm{~mL}$ of methylene chloride, and the appropriate ligand was added if required. The resulting source of palladium(0) was diluted with $10 \mathrm{~mL}$ of methylene chloride and the ruthenium catalyst was introduced, followed by allyl acetate $(113 \mu \mathrm{L}, 1.05 \mathrm{mmol}, 1.05$ equiv.). In another flask, the appropriate monosubstituted malonate $(1 \mathrm{mmol}, 1$ equiv.) dissolved in $6 \mathrm{~mL}$ of methylene chloride was deprotonated with sodium hydride (44 mg, $1.1 \mathrm{mmol}, 1.1$ equiv., $60 \%$ in mineral oil) before being added via cannula to the solution containing the catalytic systems. The resulting mixture was refluxed for $1 \mathrm{~h}$, and then allowed to cool down to room temperature. The reaction was quenched with a saturated solution of $\mathrm{NH}_{4} \mathrm{Cl}$. $\mathrm{The}$ organic layer was separated and the aqueous layer extracted with methylene chloride. The combined organic layers were washed with brine, dried over $\mathrm{MgSO}_{4}$ and concentrated under reduced pressure. The crude product was purified by flash chromatography on silica gel eluting with pentane / $\mathrm{Et}_{2} \mathrm{O} 90: 10$, to afford the expected pure product.

\section{"Allylic alkylation - allylic alkylation - ring-closing metathesis" PDOM sequence}

\section{$\underline{\text { Sequential addition of the catalytic systems : }}$}

Precursor (4, 7-12) (1 mmol, 1 equiv.), allyl acetate (226 $\mu$ l, $2.1 \mathrm{mmol}, 2.1$ equiv.) and sodium hydride (88 mg, $2.2 \mathrm{mmol}, 2.2$ equiv., $60 \%$ in mineral oil) were successively added to a solution of tetrakis(triphenylphosphine)palladium (29 mg, $25 \mu \mathrm{mol}, 2.5 \mathrm{~mol} \%$ ) in $17 \mathrm{~mL}$ of methylene chloride. The reaction mixture was stirred at room temperature until disappearance of the starting material (TLC monitoring). Grubbs' catalyst $2^{\text {nd }}$ generation ( $64 \mathrm{mg}, 75 \mu \mathrm{mol}, 7.5 \mathrm{~mol} \%$ ) was then introduced and the resulting mixture was refluxed for $4 \mathrm{~h}$. After cooling to room temperature, the reaction was quenched with a saturated solution of $\mathrm{NH}_{4} \mathrm{Cl}$. The organic layer was separated and the aqueous layer was extracted with methylene chloride. The combined organic layers were washed with brine, dried over $\mathrm{MgSO}_{4}$ and concentrated under reduced pressure. 
The crude product was purified by flash chromatography on silica gel eluting with cyclohexane / AcOEt to afford the expected product (2a, 13-18).

\section{$\underline{\text { Real pseudo-domino reaction : }}$}

Allyl acetate (226 $\mu \mathrm{l}, 2.1 \mathrm{mmol}, 2.1$ equiv.), dimethyl malonate (114 $\mu \mathrm{L}, 1 \mathrm{mmol}, 1$ equiv.), sodium hydride ( $88 \mathrm{mg}, 2.2 \mathrm{mmol}, 2.2$ equiv., 60\% in mineral oil) and Grubbs' catalyst $2^{\text {nd }}$ generation $(64 \mathrm{mg}, 75 \mu \mathrm{mol}$, $7.5 \mathrm{~mol} \%$ ) were successively added to a solution of tetrakis(triphenylphosphine)palladium (29 mg, $25 \mu \mathrm{mol}, 2.5$ mol \%) in $17 \mathrm{~mL}$ of methylene chloride. The resulting mixture was refluxed for $12 \mathrm{~h}$. After cooling down to room temperature, the reaction was quenched with a saturated solution of $\mathrm{NH}_{4} \mathrm{Cl}$. The organic layer was separated and the aqueous layer extracted with methylene chloride. The combined organic layers were washed with brine, dried over $\mathrm{MgSO}_{4}$ and concentrated under reduced pressure. The crude product was purified by flash chromatography on silica gel eluting with pentane / AcOEt $95: 5$ to afford $83 \mathrm{mg}(45 \%)$ of the expected product $\mathbf{2 a}$ as a bright yellow oil.

\section{III.2. Typical procedure for the allylic alkylation with ruthenium}

Sodium hydride (42 mg, $1.05 \mathrm{mmol}, 1.05$ equiv., 60\% in mineral oil), Grubbs'catalyst (62 mg, $75 \mu \mathrm{mol}$, $7.5 \mathrm{~mol} \%)$ and allyl acetate (108 $\mu \mathrm{L}, 1 \mathrm{mmol}, 1$ equiv.) were successively added to a solution of the appropriate malonate 1a or 4 (1 mmol, 1 equiv.) in freshly distilled THF $(5 \mathrm{~mL})$. The resulting mixture was stirred at room temperature during $30 \mathrm{~min}$ and then refluxed during $12 \mathrm{~h}$. After cooling down to room temperature, the reaction was quenched with a saturated solution of $\mathrm{NH}_{4} \mathrm{Cl}$. The organic layer was separated and the aqueous layer extracted with diethyl ether. The combined organic layers were washed with brine, dried over $\mathrm{MgSO}_{4}$ and concentrated under reduced pressure. The crude product was purified by flash chromatography on silica gel eluting with cyclohexane / AcOEt $95: 5$ to afford the expected products 1a and/or 3.

\section{Characterization data for the reaction products}

\section{Dimethyl 2-allylmalonate 1a}<smiles>C=CCC(C(=O)OC)C(=O)OC</smiles>

$\mathrm{C}_{8} \mathrm{H}_{12} \mathrm{O}_{4}$

Mol. Wt.: 172,18

See: II. Preparation of starting materials 


\section{Dimethyl cyclopent-3-ene-1,1-dicarboxylate 2a}

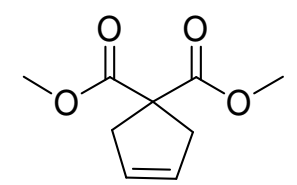

$$
\mathrm{C}_{9} \mathrm{H}_{12} \mathrm{O}_{4}
$$

Mol. Wt.: 184,19

Data in agreement with: Quinkert, G.; Schwartz, U.; Stark, H.; Weber, W.-D.; Adam, F.; Baier, H.; Frank, G.; Dürner, G. Liebigs Ann. Chem. 1982, 11, 1999-2040. Commercially available.

${ }^{1} \mathbf{H}$ NMR $\left(\mathbf{C D C l}_{3}, 400 \mathrm{MHz}\right): \delta=3.03(\mathrm{~s}, 4 \mathrm{H}), 3.75$ (s, 6H), 5.62 (s, 2H).

${ }^{13} \mathrm{C}$ NMR $\left(\mathrm{CDCl}_{3}, \mathbf{1 0 0} \mathrm{MHz}\right): \delta=41.1,53.0,58.9,128.0,172.8$.

IR (ATR) : 2954, 1731, 1624, $1434 \mathrm{~cm}^{-1}$

\section{Dimethyl cyclohex-3-ene-1,1-dicarboxylate 2b}<smiles>COC(=O)C1(C(=O)OC)CC=CCC1</smiles>

$$
\mathrm{C}_{10} \mathrm{H}_{14} \mathrm{O}_{4}
$$

Mol. Wt.: 198,22

Data in agreement with: Snider, B. B.; Merritt, J. E.; Dombroski, M. A.; Buckman, B. O. J. Org. Chem. 1991, 56, 5544-5553.

${ }^{1} \mathbf{H}$ NMR $\left(\mathbf{C D C l}_{3}, \mathbf{4 0 0} \mathbf{M H z}\right): \delta=2.07-2.16(\mathrm{~m}, 4 \mathrm{H}), 2.55(\mathrm{~m}, 2 \mathrm{H}), 3.72(\mathrm{~s}, 6 \mathrm{H}), 5.66(\mathrm{~m}, 2 \mathrm{H})$.

${ }^{13} \mathbf{C ~ N M R}\left(\mathbf{C D C l}_{3}, \mathbf{1 0 0} \mathbf{M H z}\right): \delta=22.4,27.6,30.6,52.7,53.0,124.0,126.2,172.2$.

IR (ATR) : 2954, 1731, $1434 \mathrm{~cm}^{-1}$

\section{Dimethyl cyclohept-3-ene-1,1-dicarboxylate 2c}

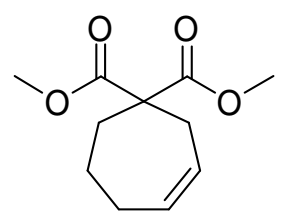

$$
\mathrm{C}_{11} \mathrm{H}_{16} \mathrm{O}_{4}
$$

Mol. Wt.: 212,24

${ }^{1} \mathbf{H}$ NMR $\left(\mathbf{C D C l}_{3}, 400 \mathrm{MHz}\right): \delta=1.61-1.67(\mathrm{~m}, 2 \mathrm{H}), 2.16(\mathrm{q}, J=5.5 \mathrm{~Hz}, 2 \mathrm{H}), 2.23-2.26(\mathrm{~m}, 2 \mathrm{H}), 2.68(\mathrm{~d}, J$ $=6.5 \mathrm{~Hz}, 2 \mathrm{H}), 3.71(\mathrm{~s}, 6 \mathrm{H}), 5.65-5.71(\mathrm{~m}, 1 \mathrm{H}), 5.83-5.89(\mathrm{~m}, 1 \mathrm{H})$.

${ }^{13} \mathbf{C}$ NMR $\left(\mathbf{C D C l}_{3}, \mathbf{1 0 0} \mathbf{M H z}\right): \delta=22.9,28.4,32.5,36.9,52.6,56.3,127.2,134.4,172.4$.

IR (ATR) : 2952, 1730, $1434 \mathrm{~cm}^{-1}$

HRMS $m / z$ calculated for $\mathrm{C}_{11} \mathrm{H}_{16} \mathrm{O}_{4} \mathrm{Na}\left(\mathrm{M}^{+}\right)$: 235.0941. Found: 235.0942 . 


\section{Dimethyl 2,2-diallylmalonate 3}<smiles>C=CCC(CC=C)(C(=O)OC)C(=O)OC</smiles>

$$
\mathrm{C}_{11} \mathrm{H}_{16} \mathrm{O}_{4}
$$

Mol. Wt.: 212,24

Data in agreement with: Hayashi, Y.; Gotoh, H.; Tamura, T.; Yamaguchi, H.; Masui, R.; Shoji, M. J. Am. Chem. Soc. 2005, 127, 16028 - 16029. Commercially available.

${ }^{1} \mathbf{H}$ NMR $\left(\mathbf{C D C l}_{3}, \mathbf{4 0 0} \mathbf{M H z}\right): \delta=2.64(\mathrm{dd}, J=1.0,6.5 \mathrm{~Hz}, 4 \mathrm{H}), 3.72(\mathrm{~s}, 6 \mathrm{H}), 5.09-5.13(\mathrm{~m}, 4 \mathrm{H}), 5.59-5.70$ $(\mathrm{m}, 2 \mathrm{H})$.

${ }^{13} \mathrm{C} \mathrm{NMR}\left(\mathrm{CDCl}_{3}, \mathbf{1 0 0} \mathbf{M H z}\right): \delta=37.0,52.5,57.8,119.4,132.3,171.3$.

\section{1,1-diacetylcyclopent-3-ene 13}

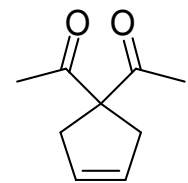

$$
\mathrm{C}_{9} \mathrm{H}_{12} \mathrm{O}_{2}
$$

Mol. Wt.: 152,19

Data in agreement with: Bose, G.; Ullah, E.; Langer, P. Chem. Eur. J. 2004, 10, 6015-6028.

${ }^{1} \mathrm{H}$ NMR $\left(\mathrm{CDCl}_{3}, 400 \mathrm{MHz}\right): \delta=2.13$ (s, 6H), 2.89 (s, 4H), 5.57 (s, 2H).

${ }^{13} \mathbf{C} \mathbf{N M R}\left(\mathbf{C D C l}_{3}, \mathbf{1 0 0} \mathbf{M H z}\right): \delta=.26 .5,37.8,73.3,128.0,205.1$.

IR (ATR) : 2923, 1696, 1626, 1431, $1356 \mathrm{~cm}^{-1}$

\section{Methyl 1-acetyl-cyclopent-3-enecarboxylate 14}

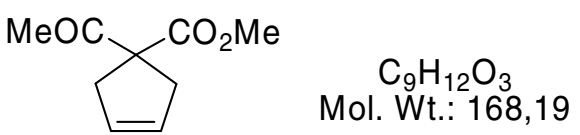

Data in agreement with:. Deprès, J.-P.; Greene, A. P. J. Org. Chem. 1984, 49, 928-931.

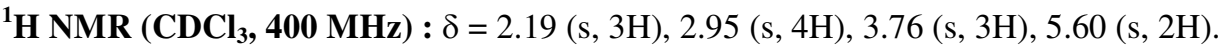

${ }^{13} \mathbf{C ~ N M R}\left(\mathbf{C D C l}_{3}, \mathbf{1 0 0} \mathbf{M H z}\right): \delta=26.1,39.5,52.9,65.5,127.9,173.6,202.9$.

IR (ATR) : 3063, 2928, 2854, 1744, 1714, 1435, $1358 \mathrm{~cm}^{-1}$ 


\section{Ethyl-1-nitro-cyclopent-3-enecarboxylate 15}

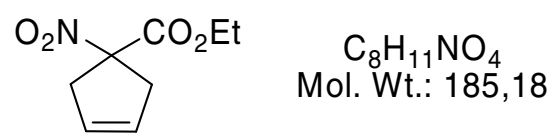

${ }^{1} \mathbf{H}$ NMR $\left(\mathbf{C D C l}_{3}, 400 \mathrm{MHz}\right): \delta=1.30\left(\mathrm{t}, 3 \mathrm{H},{ }^{3} \mathrm{~J}=7.0 \mathrm{~Hz}\right), 3.24\left(\mathrm{~d}, 2 \mathrm{H},{ }^{2} \mathrm{~J}=17 \mathrm{~Hz}\right.$, part of AB system), $3.48(\mathrm{~d}$, $2 \mathrm{H},{ }^{2} J=17 \mathrm{~Hz}$, part of AB system), $4.29\left(\mathrm{q}, 2 \mathrm{H},{ }^{3} J=7.0 \mathrm{~Hz}\right), 5.69(\mathrm{~s}, 2 \mathrm{H})$.

${ }^{13} \mathbf{C}$ NMR $\left(\mathbf{C D C l}_{3}, \mathbf{1 0 0} \mathbf{M H z}\right): \delta=13.9,42.8,63.2,98.0,127.1,167.4$

IR (ATR) : 2984, 1746, 1550, 1428, 1353, 1270, $1208 \mathrm{~cm}^{-1}$

\section{8,8-dimethyl-7,9-dioxaspiro[4.5]dec-2-ene-6,10-dione 16}

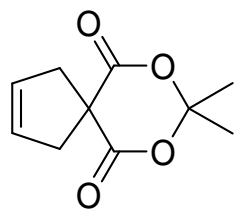

$$
\mathrm{C}_{10} \mathrm{H}_{12} \mathrm{O}_{4}
$$

Mol. Wt.: 196,2

Data in agreement with: Kotha, S.; Manivannan, E.; Ganesh, T.; Sreenivasachary, N.; Deb, A. Synlett 1999, 1618-1620.

${ }^{1} \mathrm{H}$ NMR $\left(\mathbf{C D C l}_{3}, \mathbf{4 0 0} \mathbf{M H z}\right): \delta=.1 .74(\mathrm{~s}, 6 \mathrm{H}), 3.12(\mathrm{~s}, 4 \mathrm{H}), 5.69(\mathrm{~s}, 2 \mathrm{H})$.

${ }^{13} \mathbf{C}$ NMR $\left(\mathbf{C D C l}_{3}, \mathbf{1 0 0} \mathbf{M H z}\right): \delta=29.0,46.7,50.7,104.8,127.2,170.8$.

IR (ATR) : 3062, 2928, 1743, $1666 \mathrm{~cm}^{-1}$

\section{Spiro[4.5]dec-2-ene-6,10-dione 17}

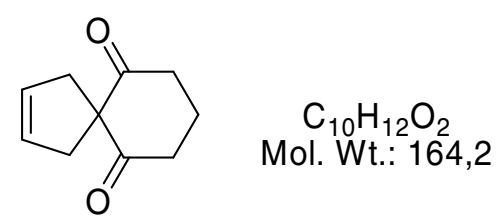

Data in agreement with: Kotha, S.; Manivannan, E. Arkivoc 2003, 67-76.

${ }^{1} \mathrm{H}$ NMR $\left(\mathrm{CDCl}_{3}, 400 \mathrm{MHz}\right.$ ) : $\delta=.1 .97$ (quint., $2 \mathrm{H},{ }^{3} J=6.5 \mathrm{~Hz}$ ), 2.69 (t, $4 \mathrm{H},{ }^{3} J=6.5 \mathrm{~Hz}$ ), 2.85 (s, 4H), 5.52 (s, $2 \mathrm{H})$.

${ }^{13} \mathbf{C} \mathbf{N M R}\left(\mathbf{C D C l}_{3}, \mathbf{1 0 0} \mathbf{M H z}\right): \delta=17.6,37.7,39.2,70.9,127.1,207.1$.

IR (ATR) : 2926, 2852, 1723, 1688, $1629 \mathrm{~cm}^{-1}$ 
7,9-dimethyl-9-diazaspiro[4.5]dec-2-ene-6,8,10-trione 18

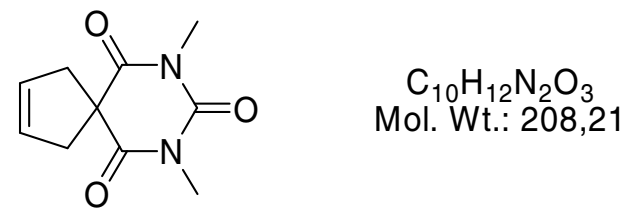

Data in agreement with: Kotha, S.; Deb, A. C.; Kumar, R. V. Bioorg. Med. Chem. 2005, 16, 1039-1043.

${ }^{1} \mathrm{H}$ NMR $\left(\mathrm{CDCl}_{3}, \mathbf{4 0 0} \mathrm{MHz}\right): \delta=.2 .97$ (s, 4H), 3.27 (s, 6H), $5.63(\mathrm{~s}, 2 \mathrm{H})$.

${ }^{13} \mathbf{C}$ NMR $\left(\mathbf{C D C l}_{3}, \mathbf{1 0 0} \mathbf{M H z}\right): \delta=.29 .0,45.5,54.6,127.4,151.4,172.5$.

IR (ATR) : 3059, 3003, 2933, 2852, 1763, $1733 \mathrm{~cm}^{-1}$

\section{V. ${ }^{1} \mathrm{H}$ and ${ }^{13} \mathrm{C}$ NMR spectra}


Dimethyl 2-allylmalonate 1a

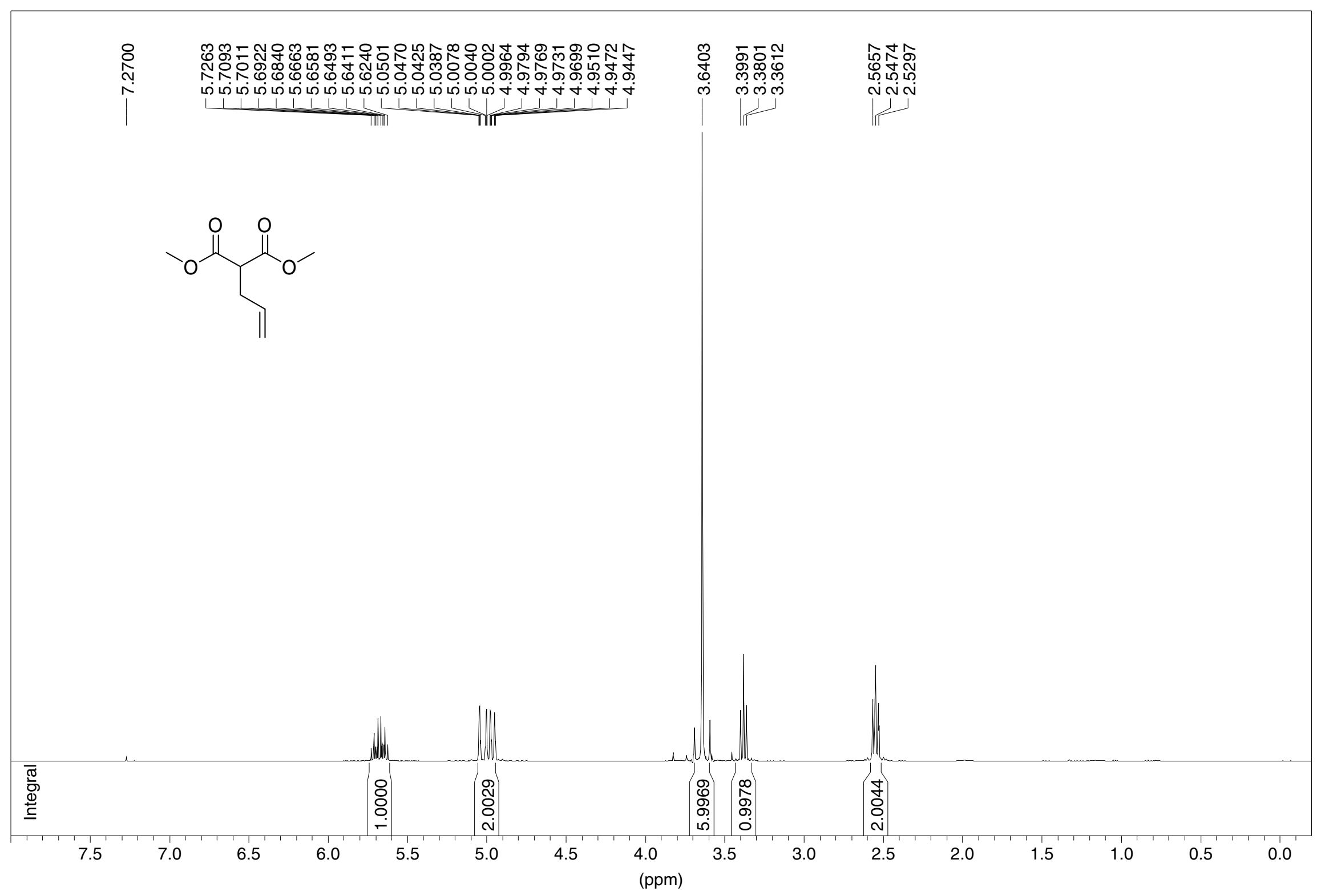


Dimethyl 2-allylmalonate 1a

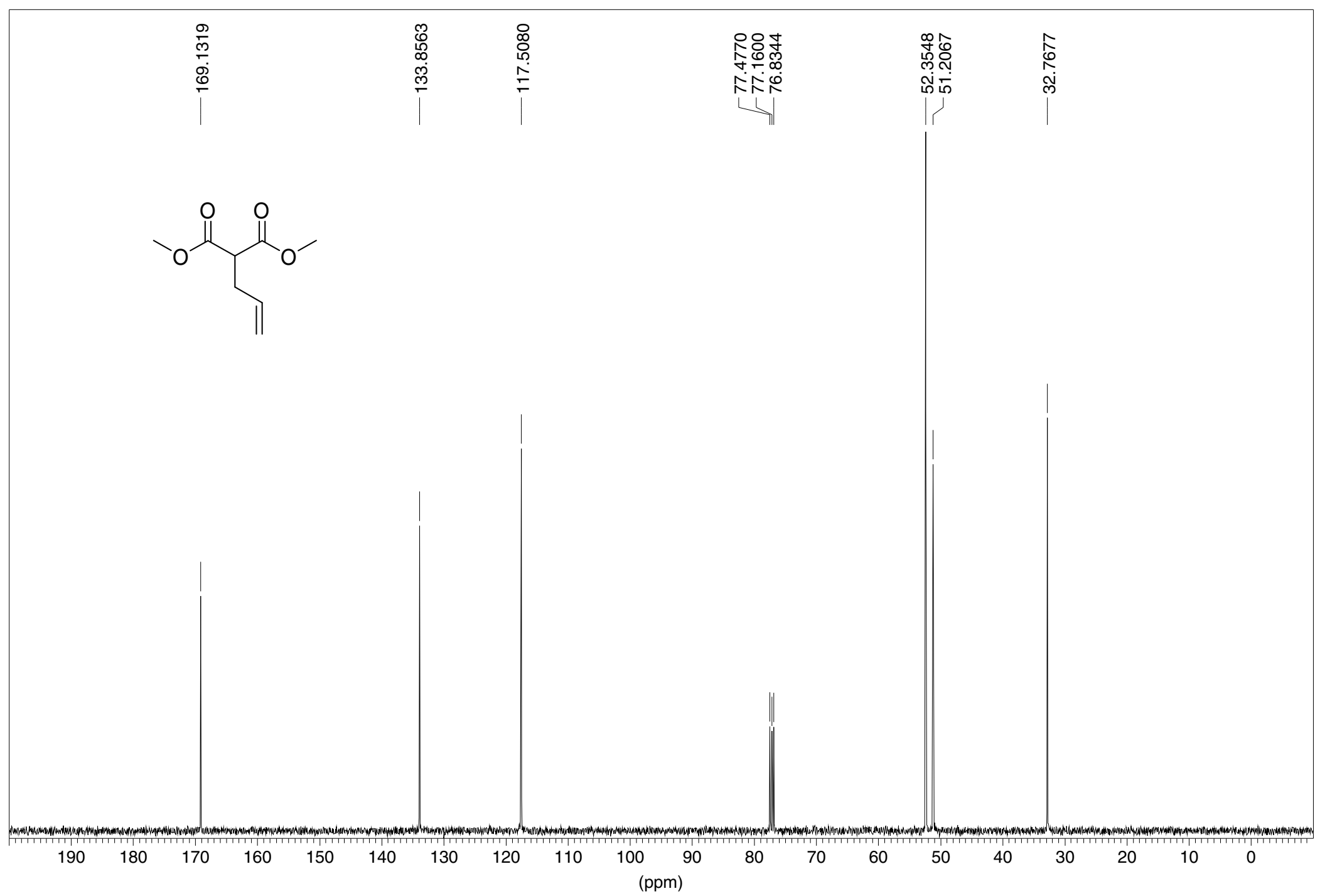


Dimethyl 2-(but-3'-enyl)malonate 1b

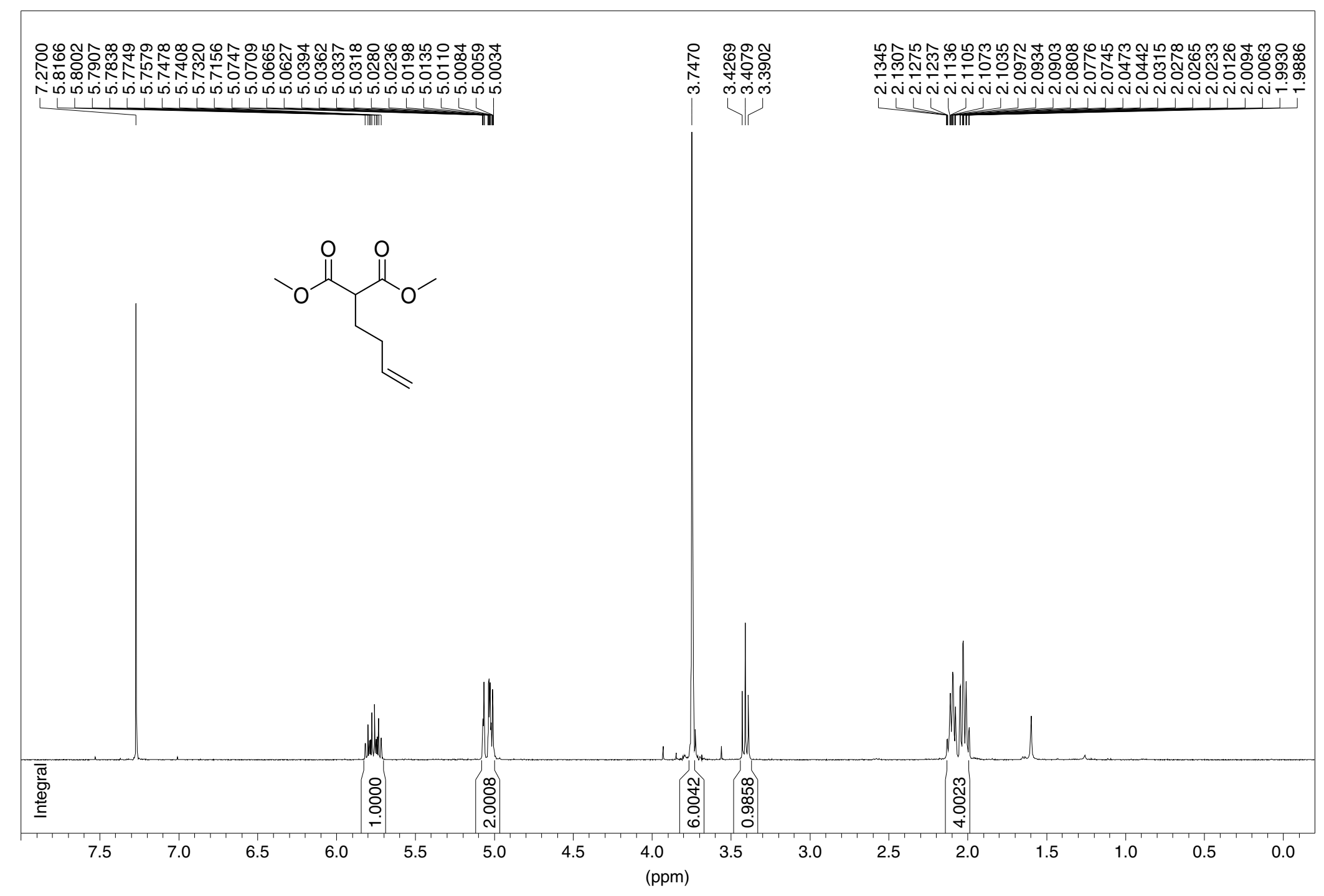




\section{Dimethyl 2-(but-3'-enyl)malonate 1b}

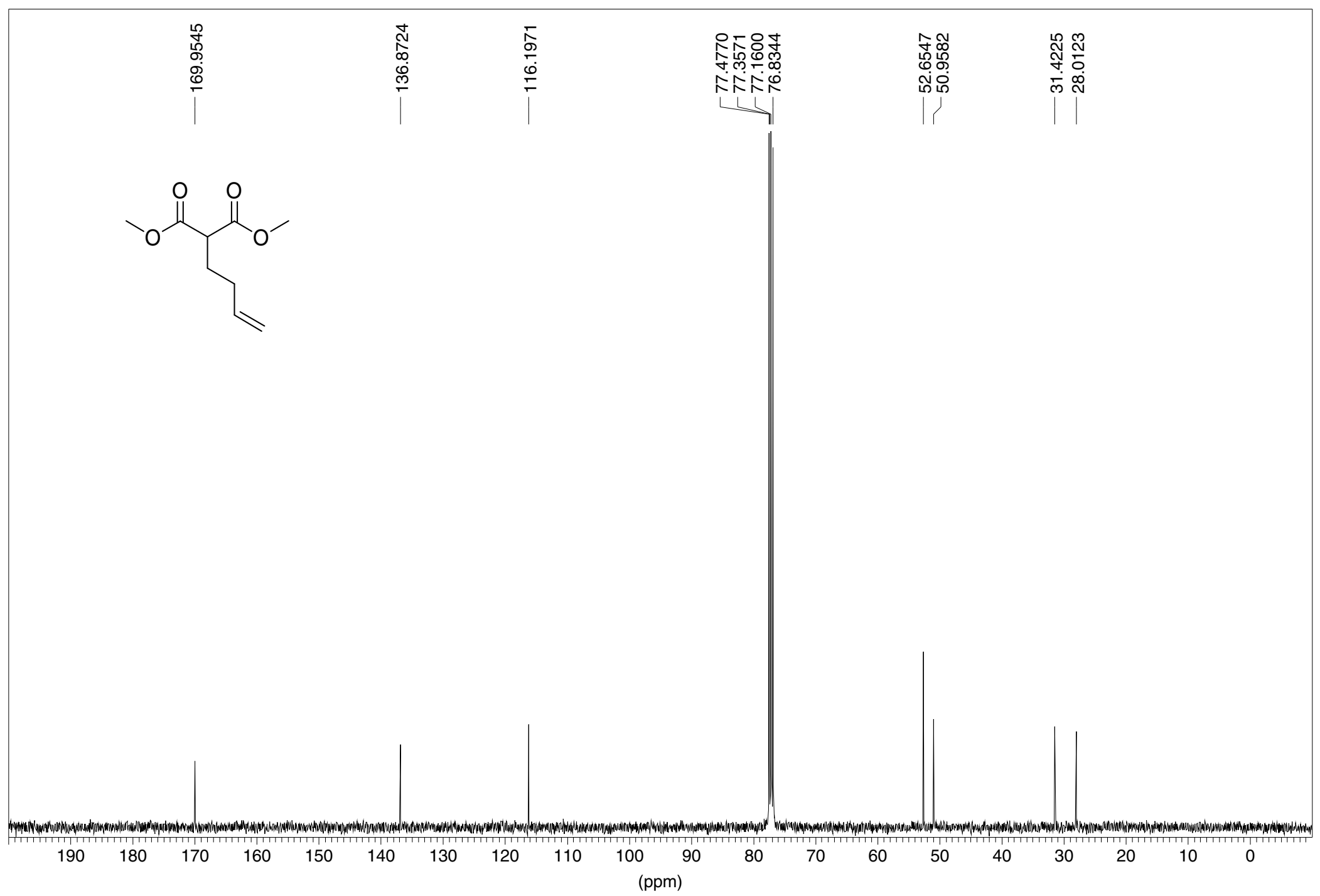


Dimethyl 2-(pent-4'-enyl)malonate 1c

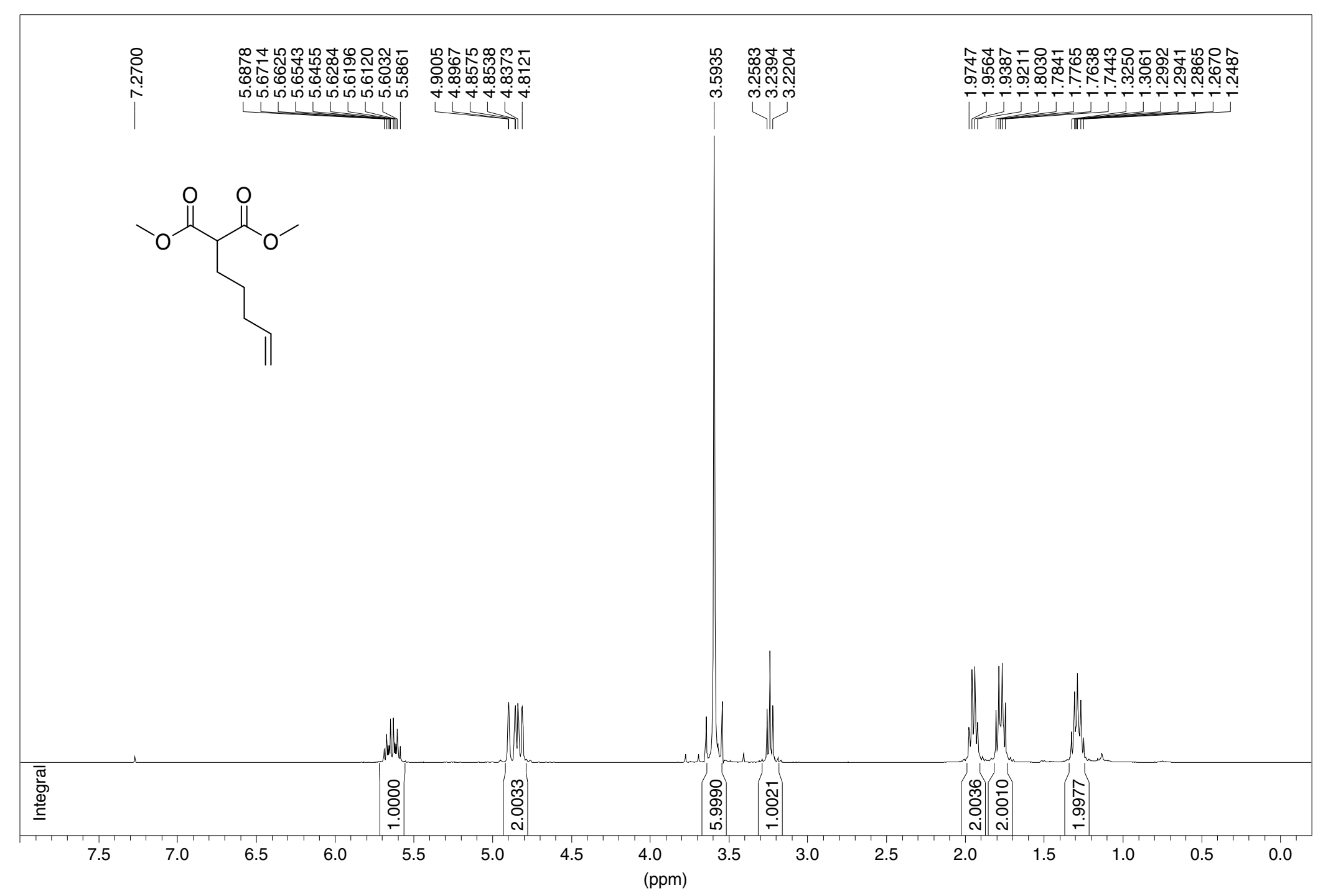


Dimethyl 2-(pent-4'-enyl)malonate 1c

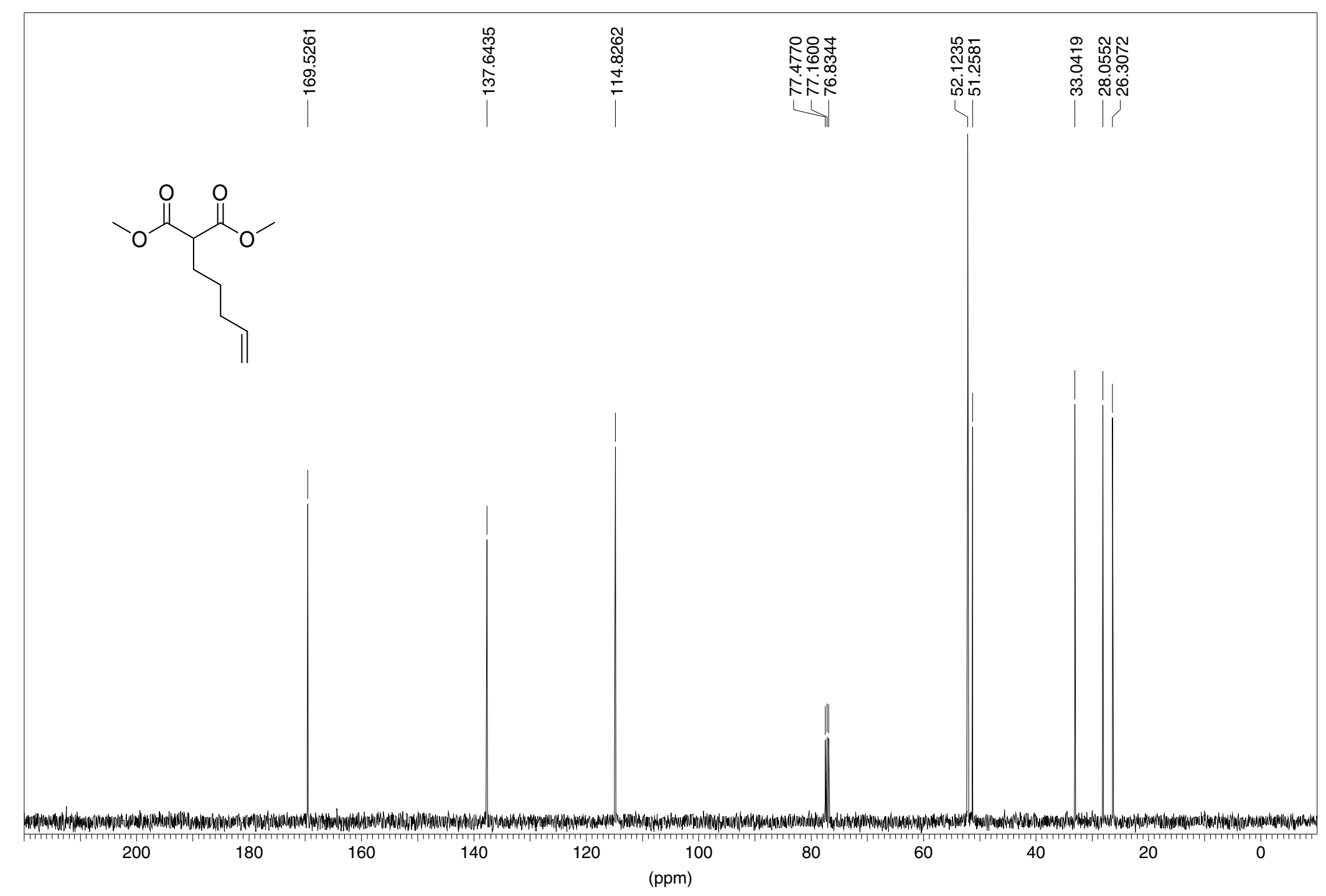


Dimethyl cyclopent-3-ene-1,1-dicarboxylate 2a

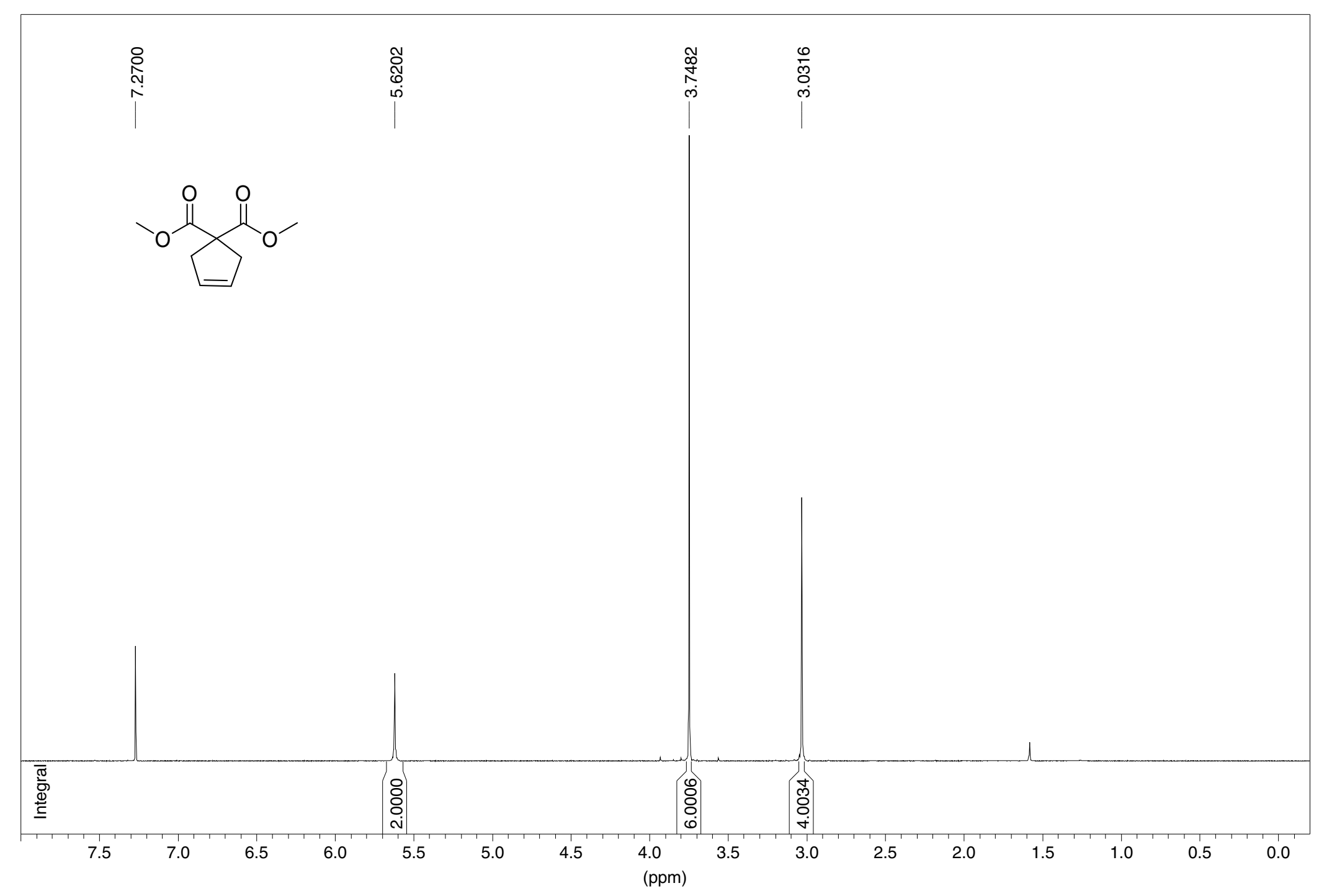


Dimethyl cyclopent-3-ene-1,1-dicarboxylate 2a

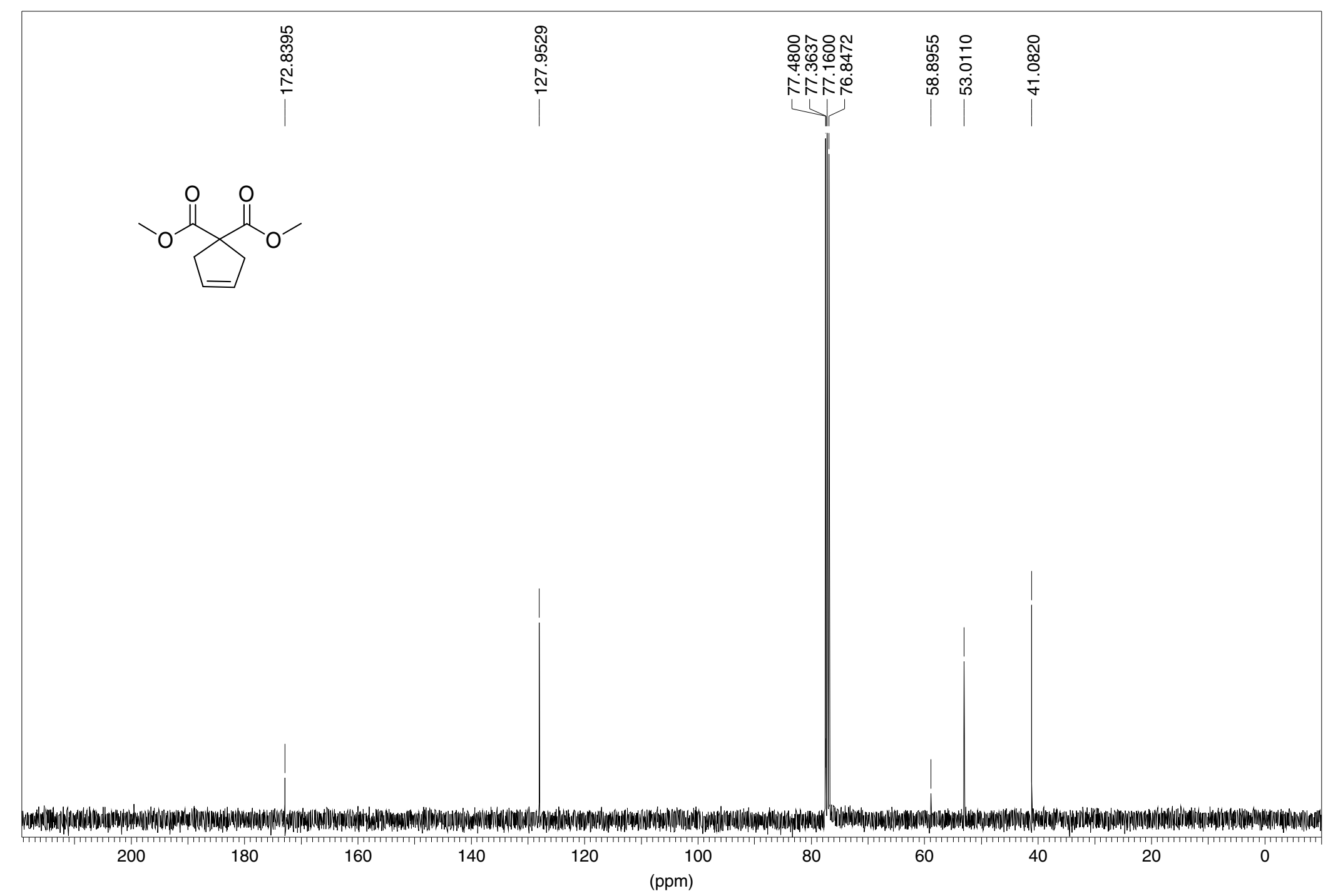


Dimethyl cyclohex-3-ene-1,1-dicarboxylate 2b

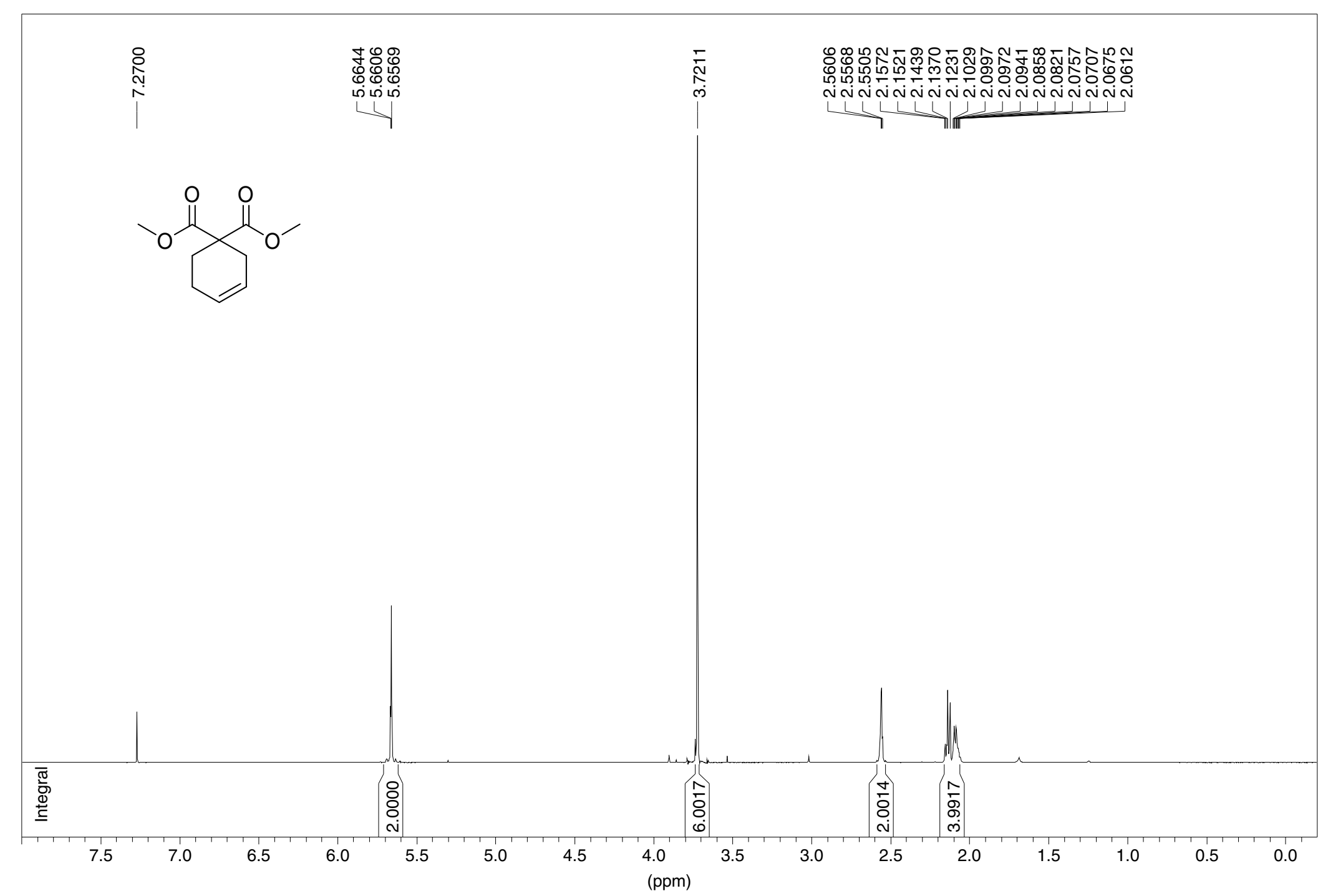


Dimethyl cyclohex-3-ene-1,1-dicarboxylate 2b

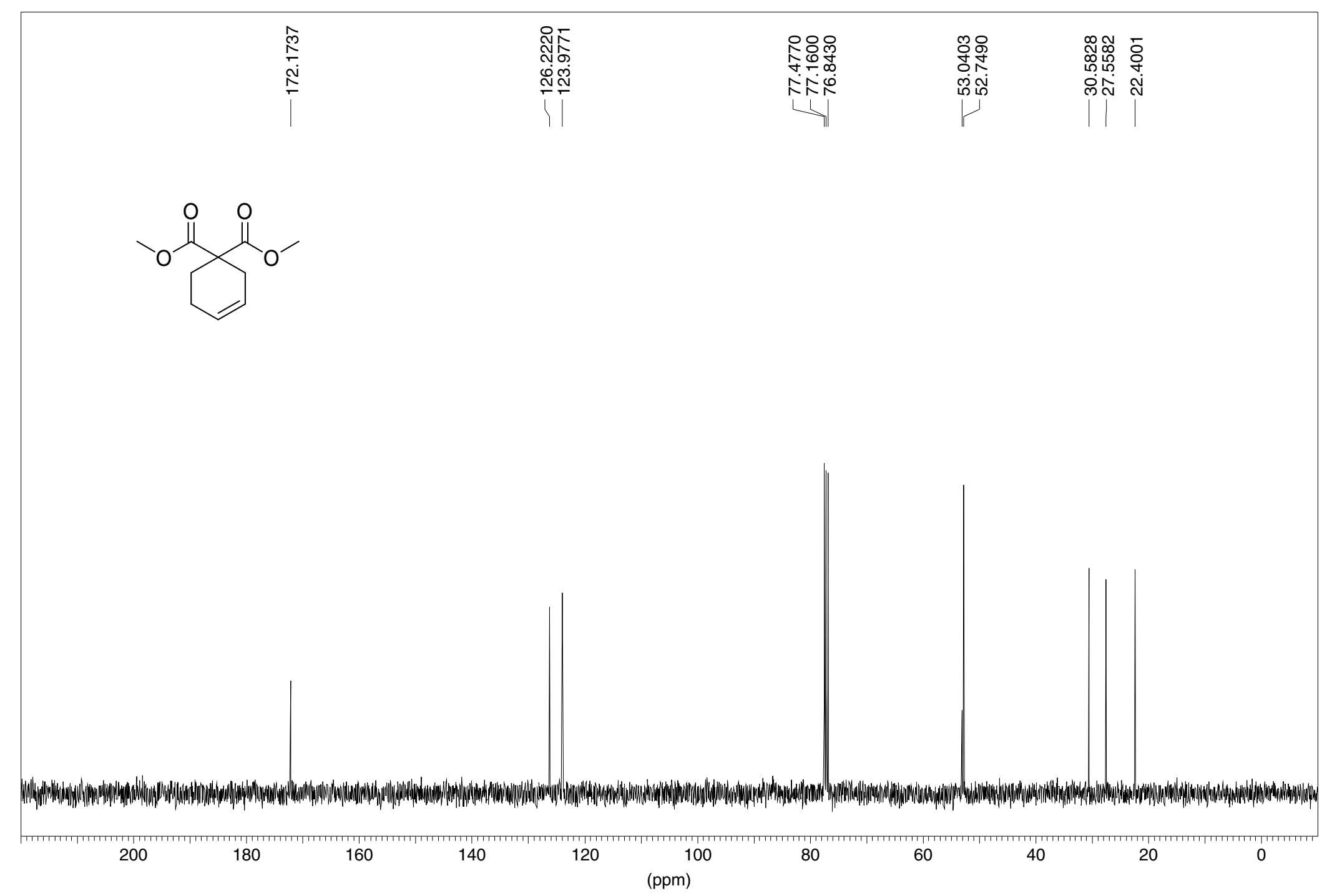


Dimethyl cyclohept-3-ene-1,1-dicarboxylate 2c

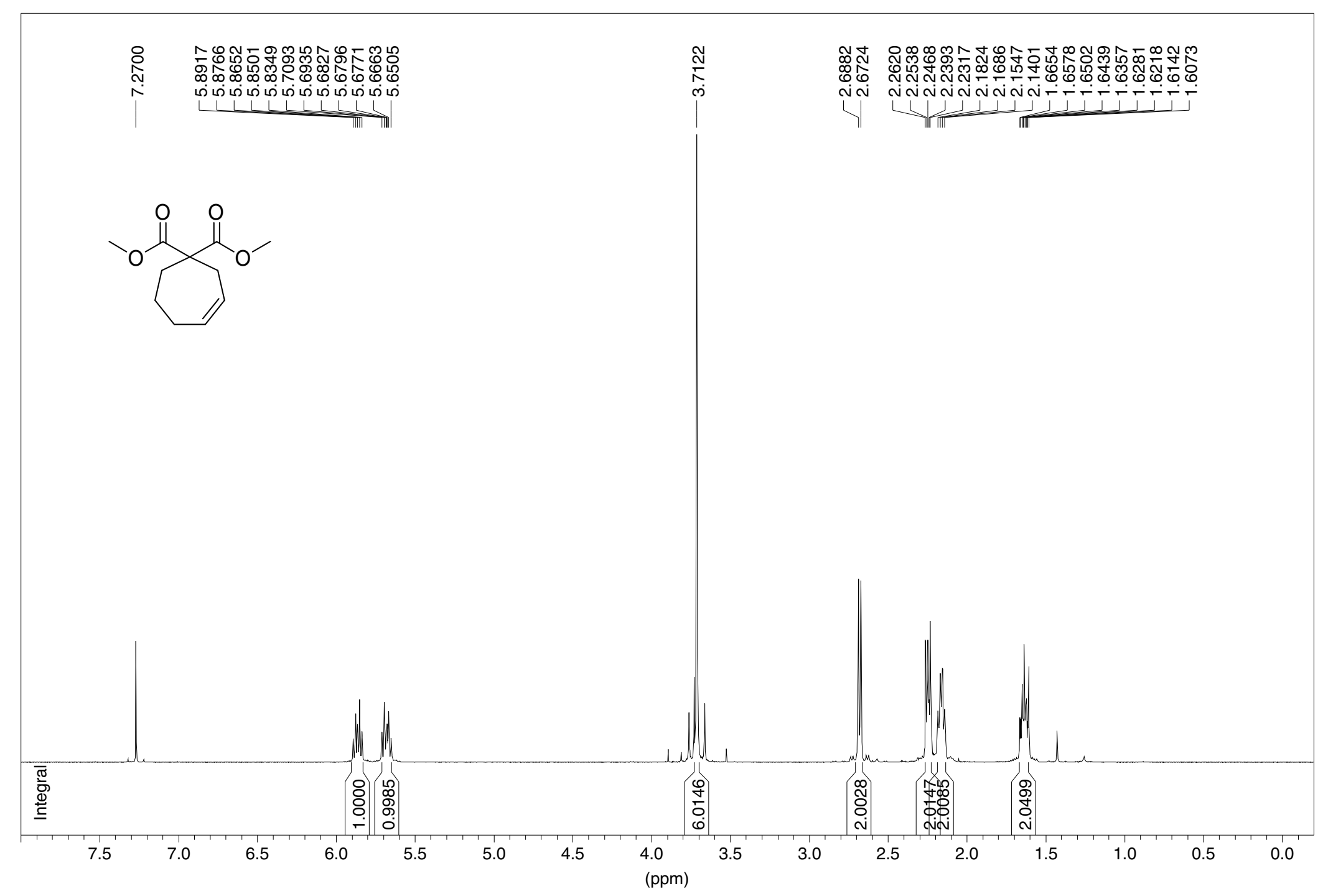


Dimethyl cyclohept-3-ene-1,1-dicarboxylate 2c

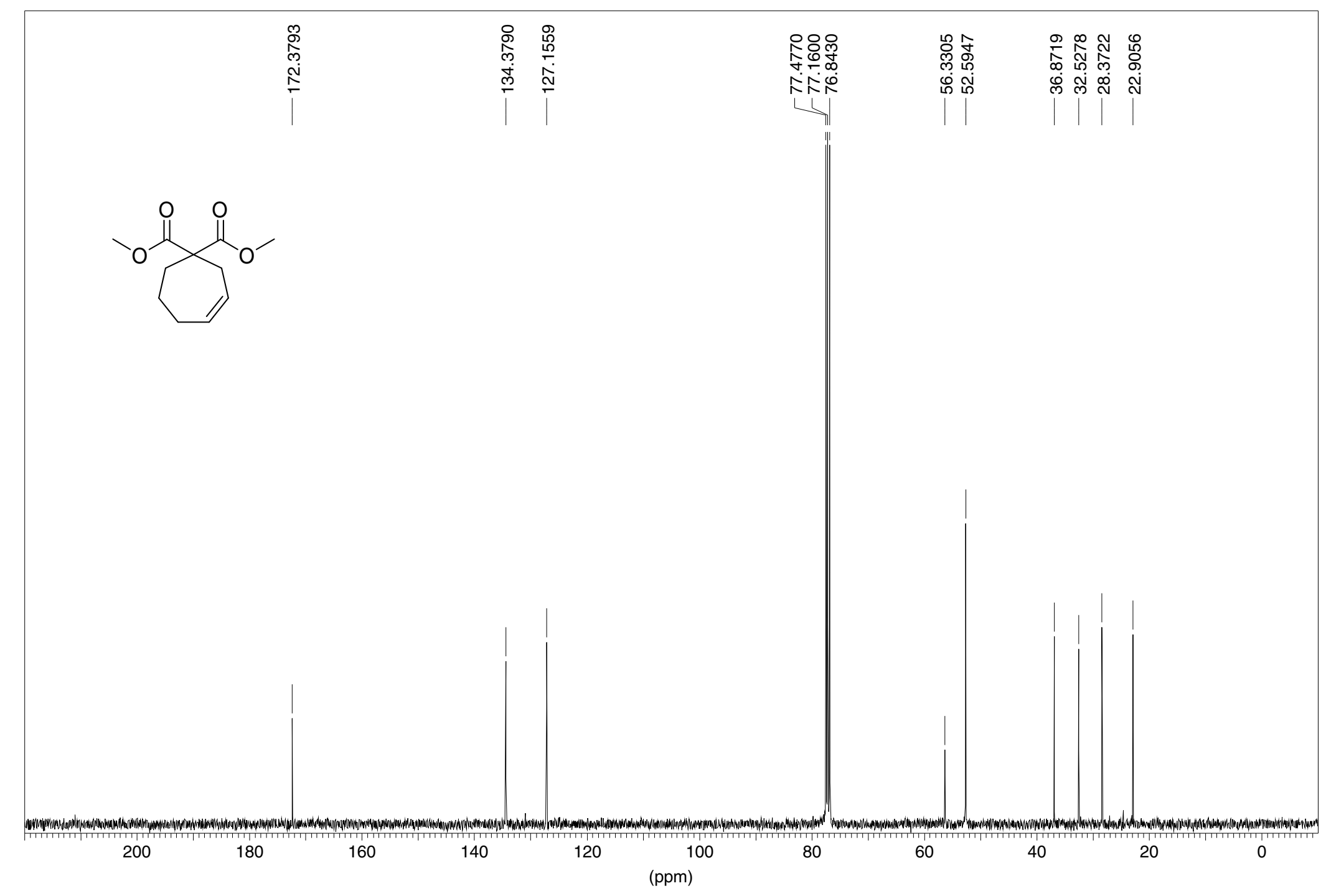


Dimethyl 2,2-diallylmalonate 3

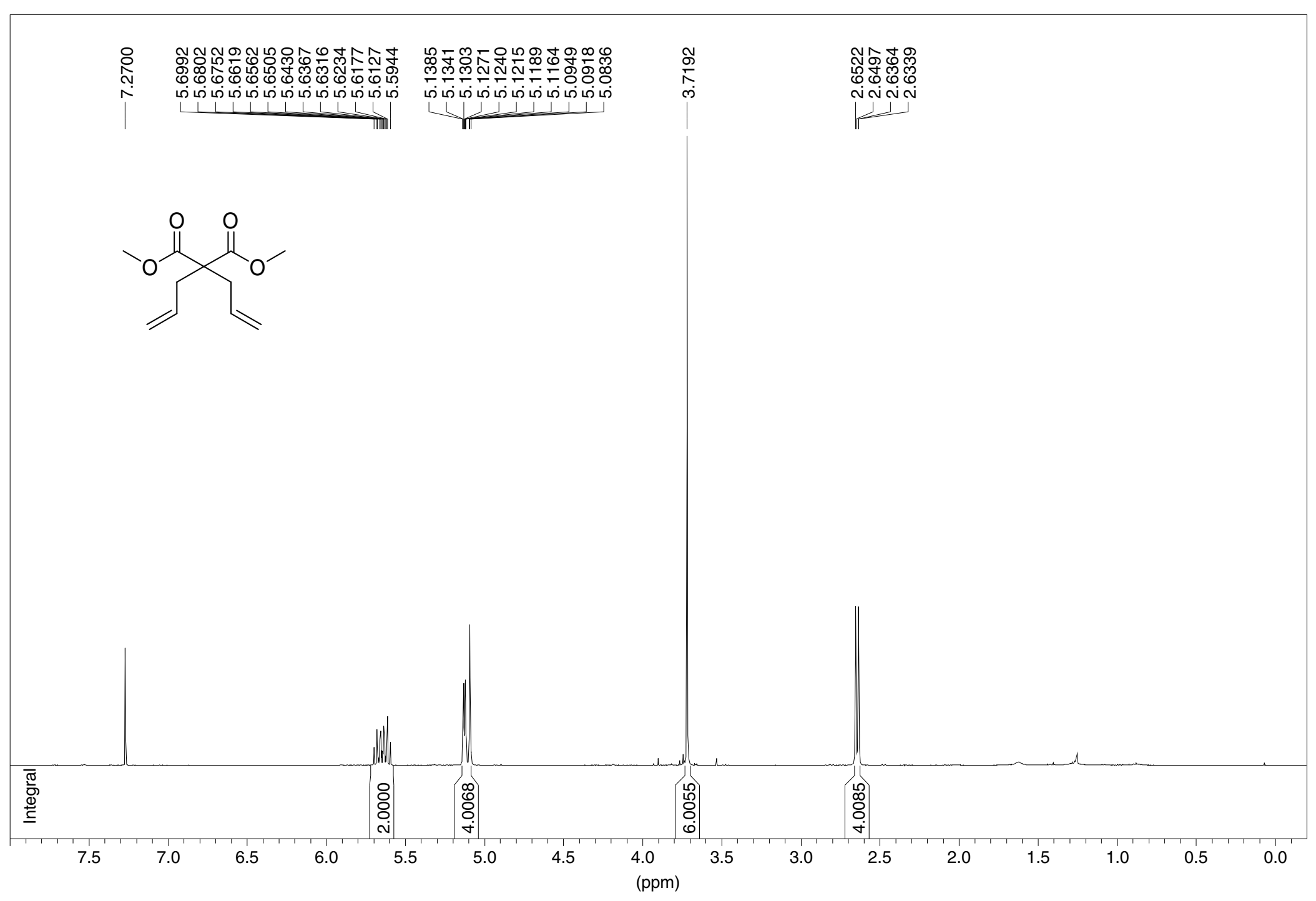


Dimethyl 2,2-diallylmalonate 3

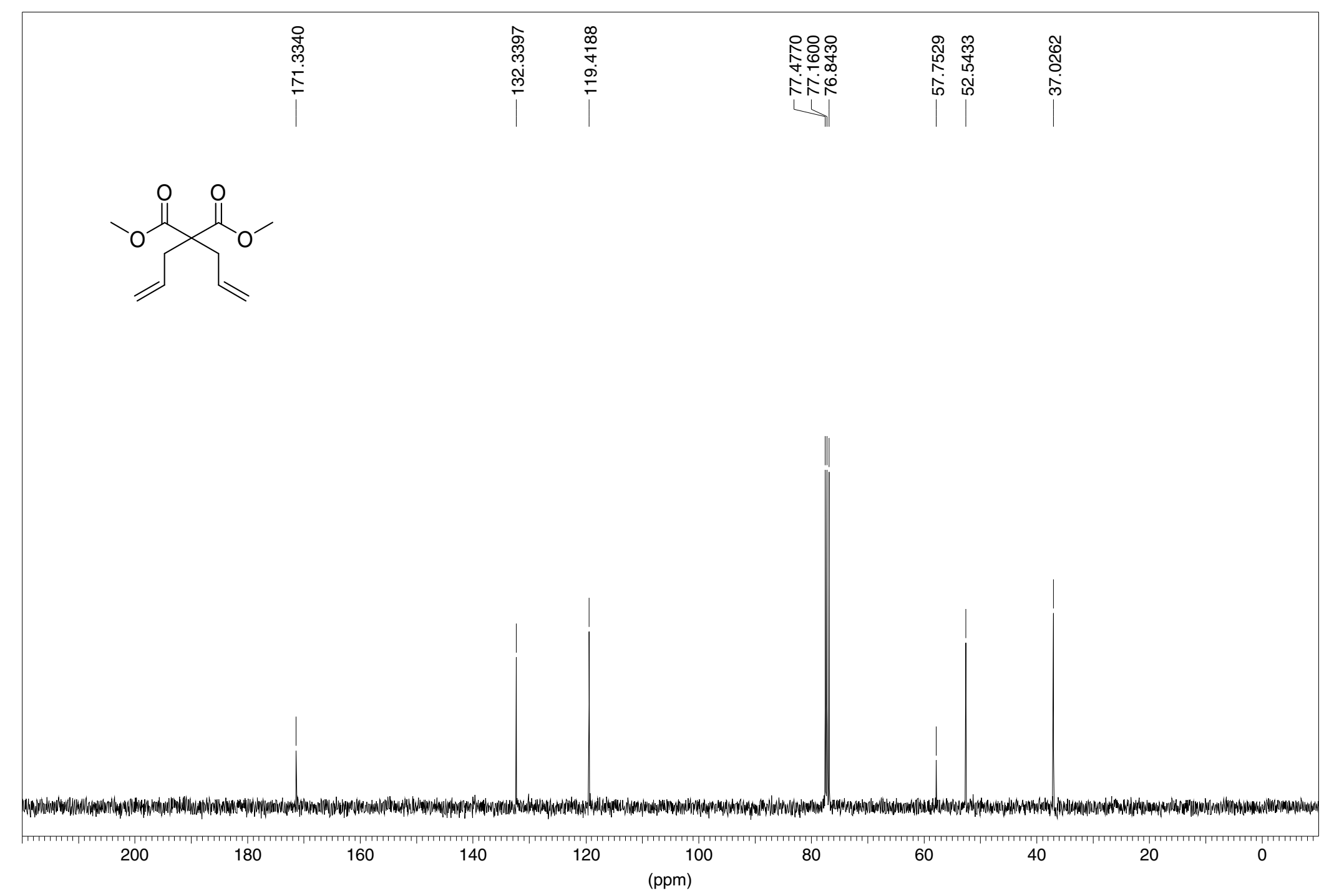




\section{1,1-diacetylcyclopent-3-ene 13}

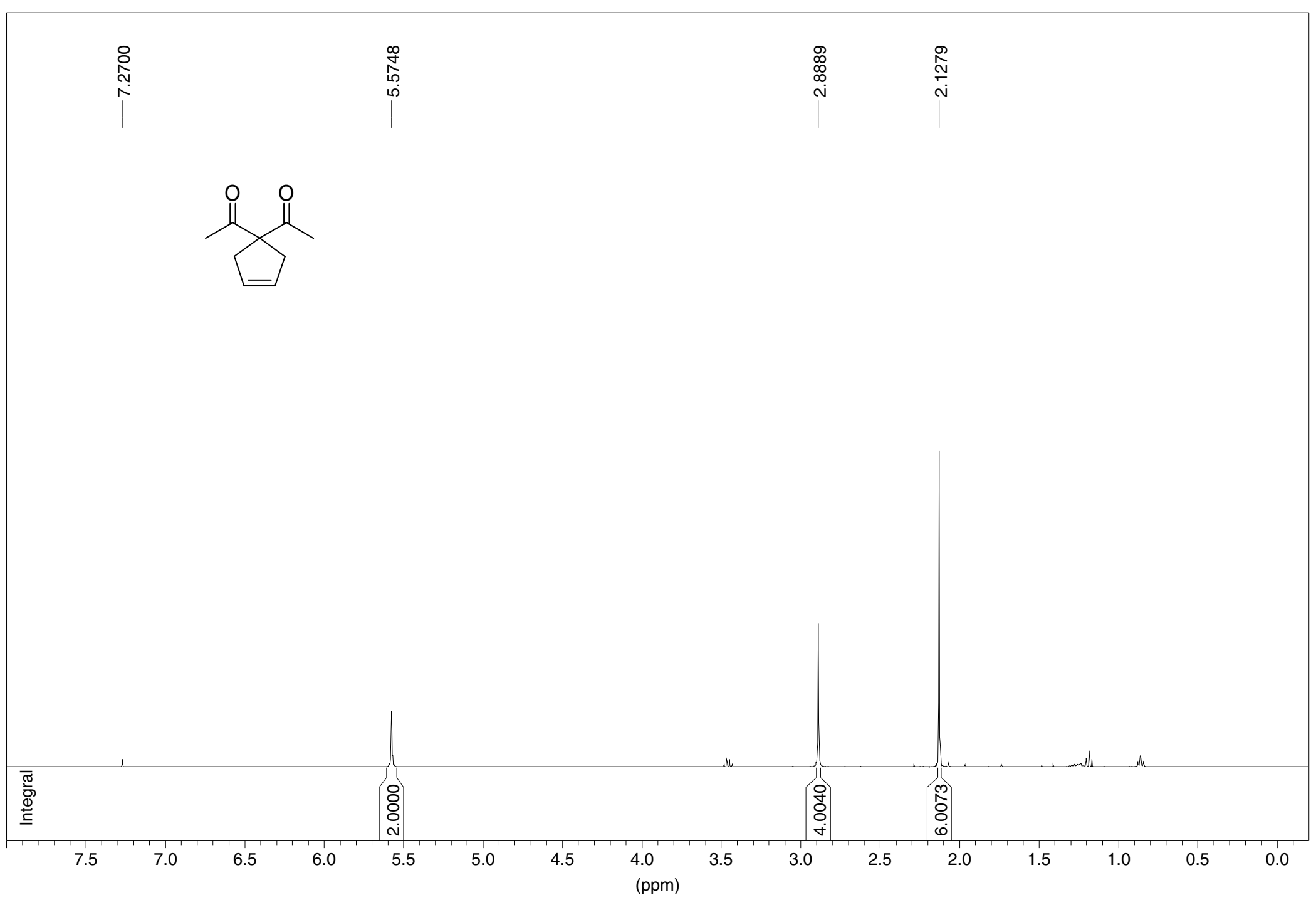




\section{1,1-diacetylcyclopent-3-ene 13}

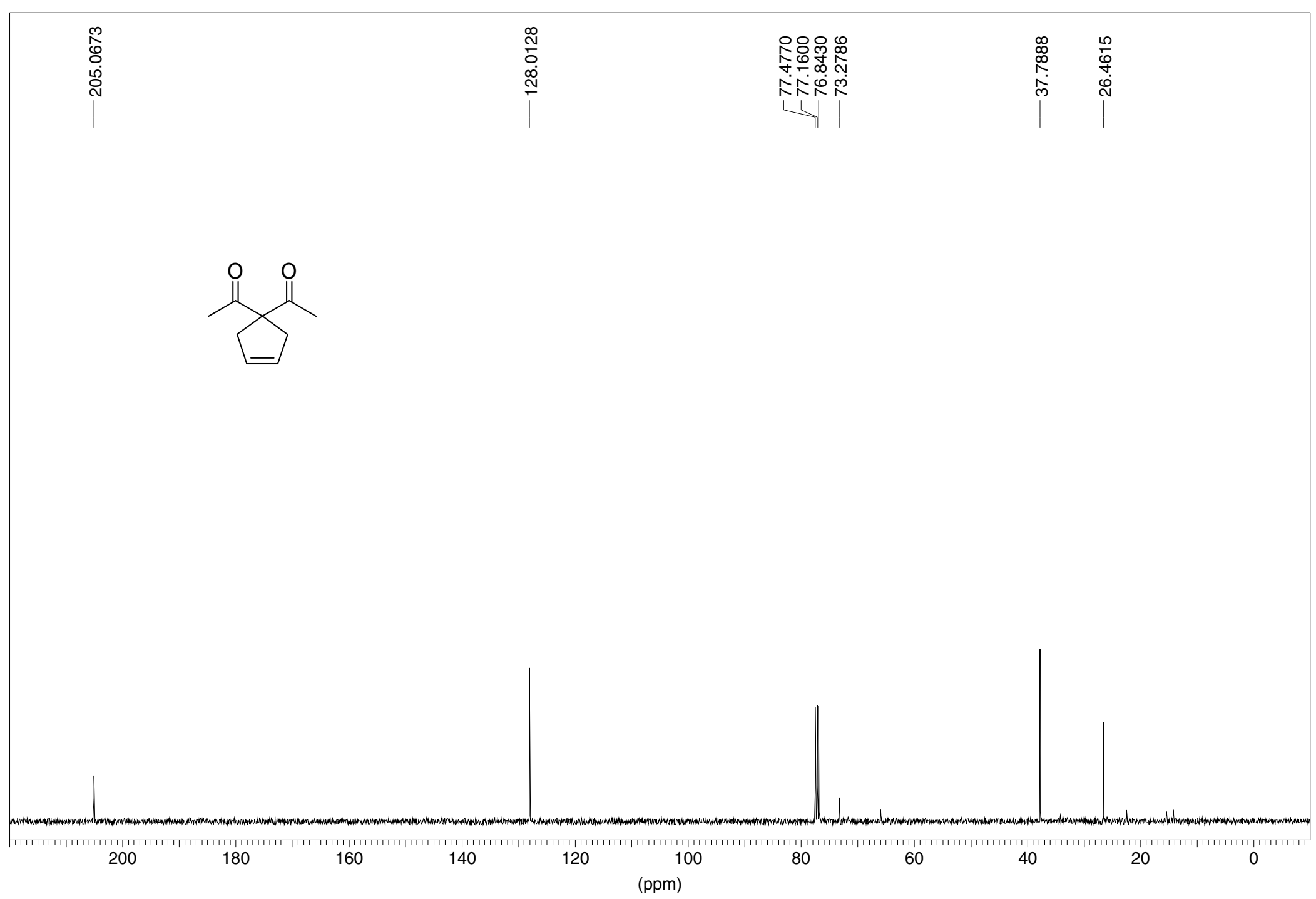


Methyl 1-acetyl-cyclopent-3-enecarboxylate 14
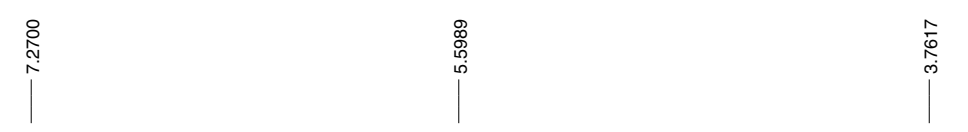

บั

|
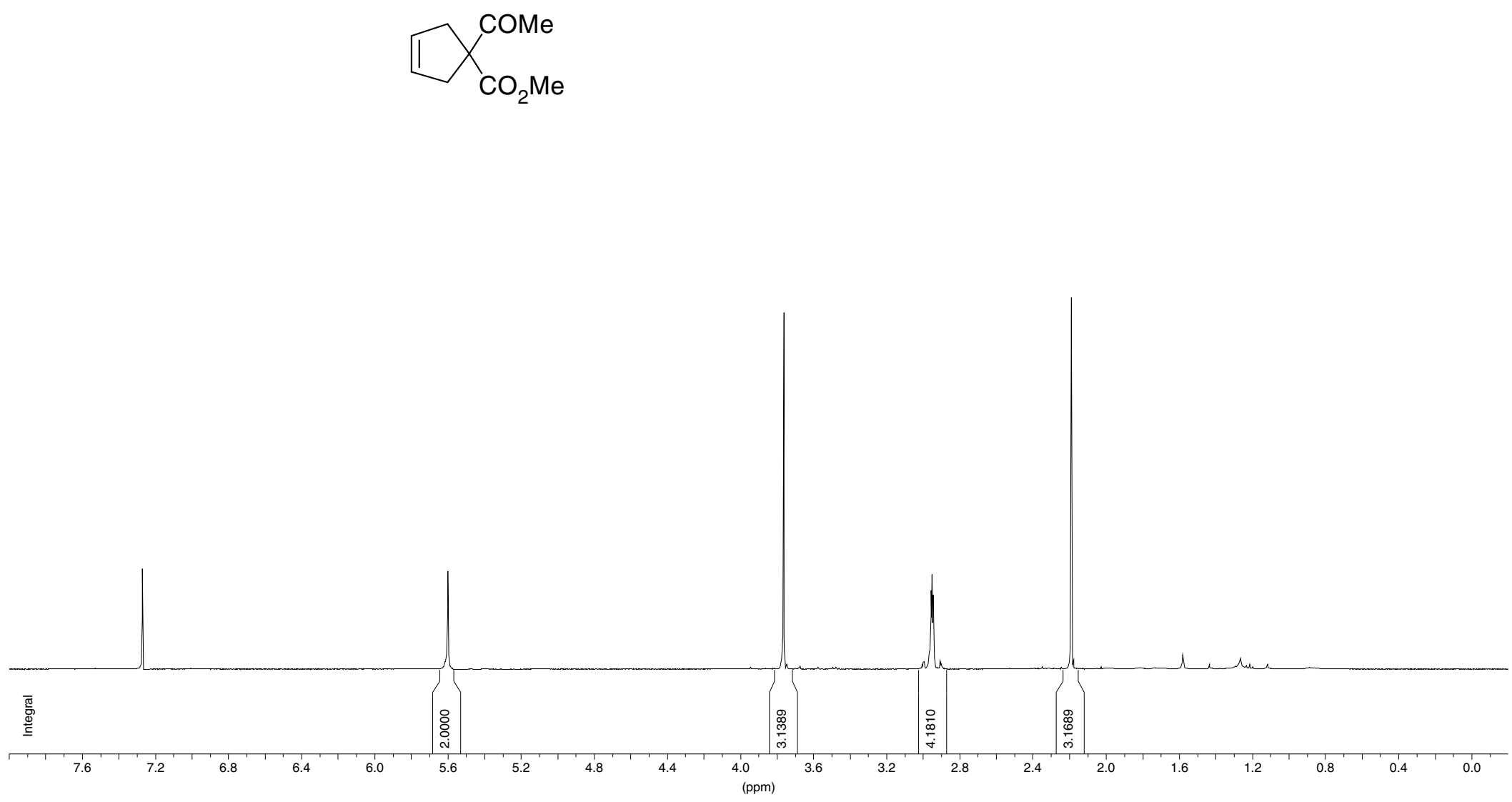

S27 
Methyl 1-acetyl-cyclopent-3-enecarboxylate 14

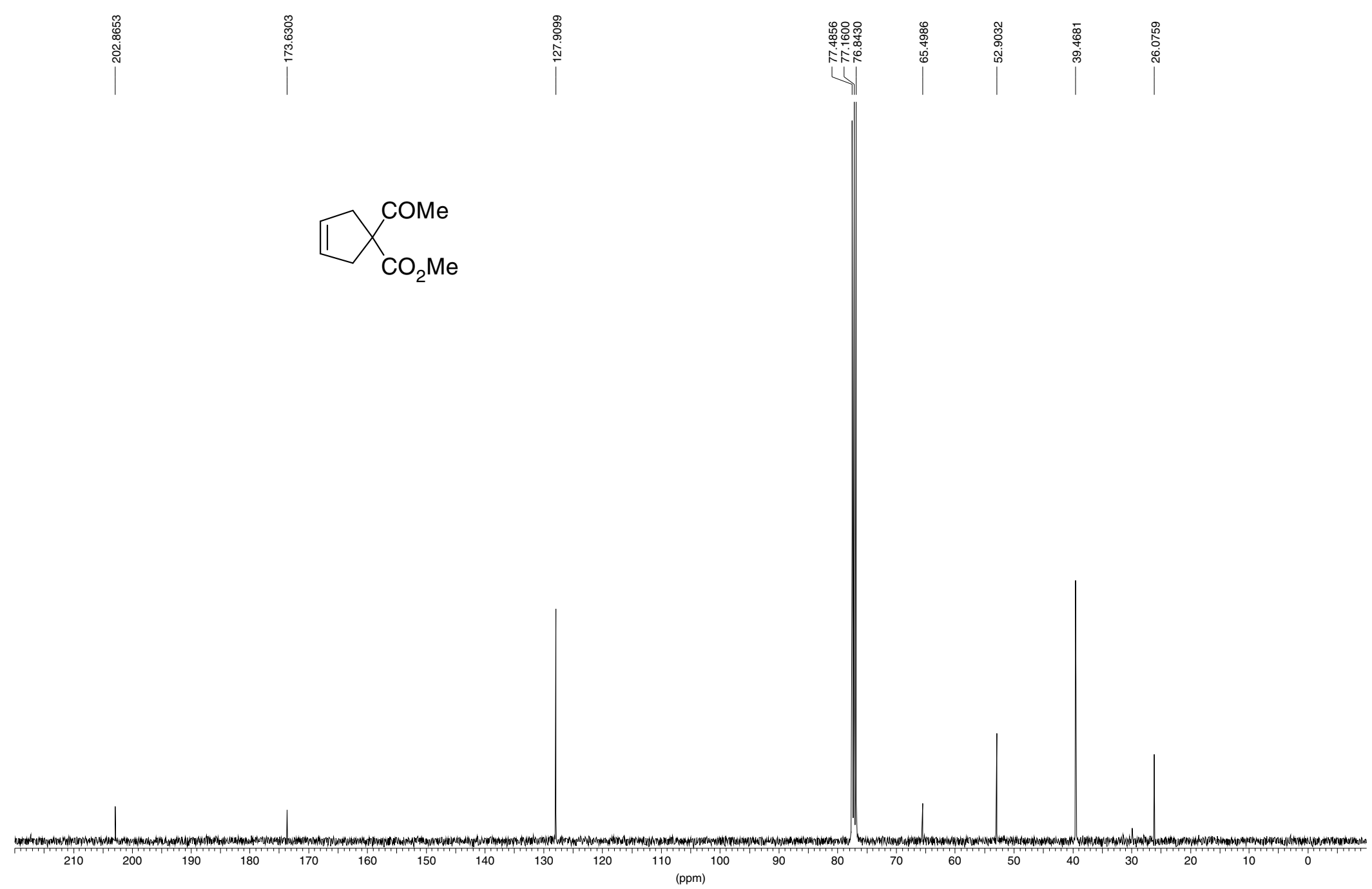


Ethyl-1-nitro-cyclopent-3-enecarboxylate 15

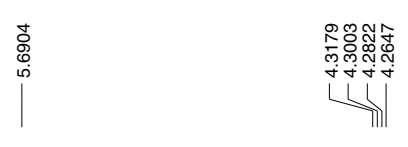

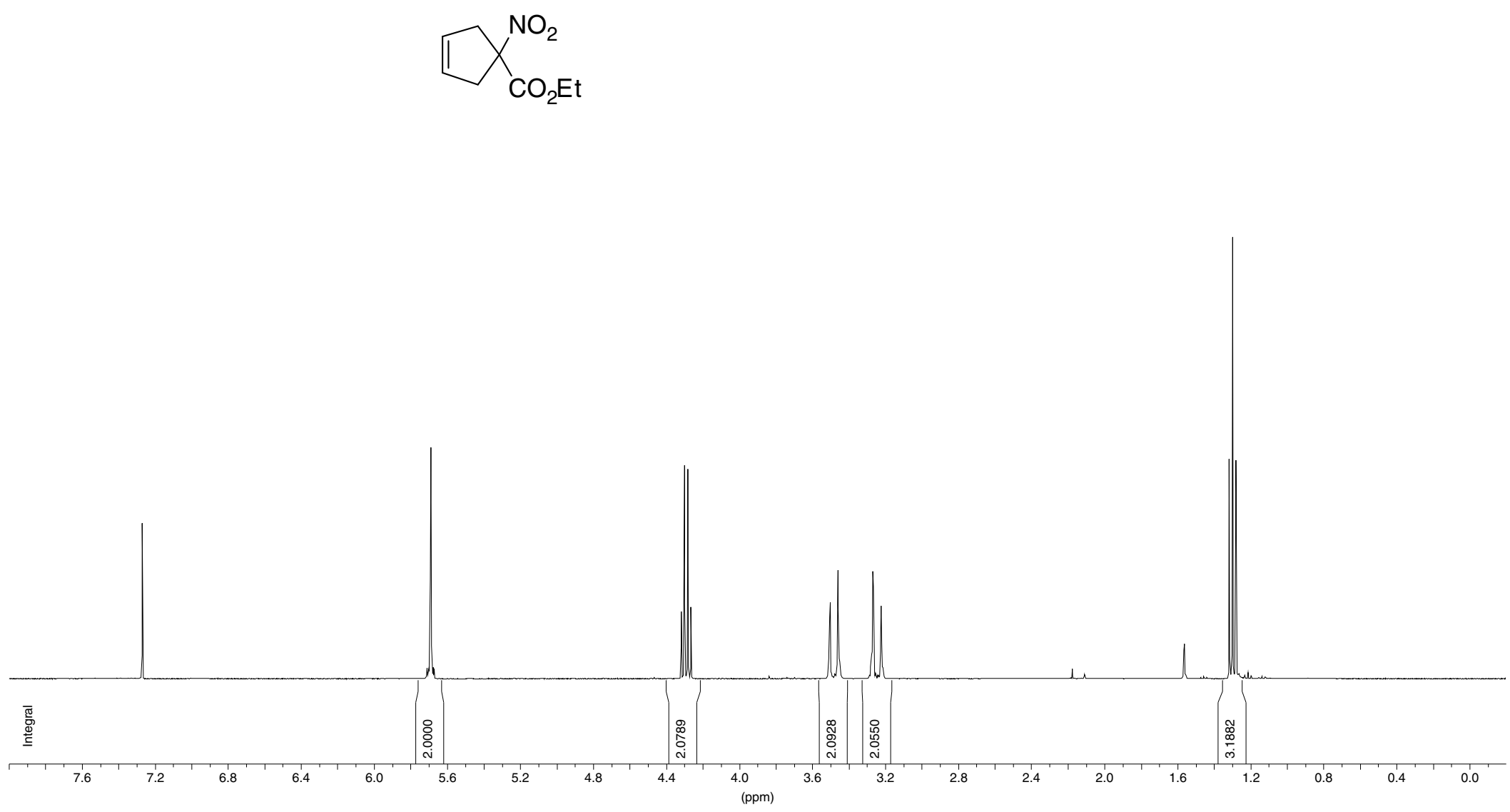

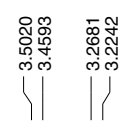

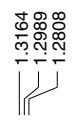

S29 
Ethyl-1-nitro-cyclopent-3-enecarboxylate 15

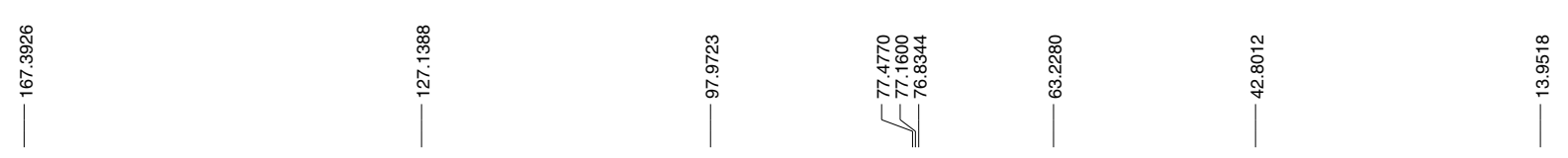

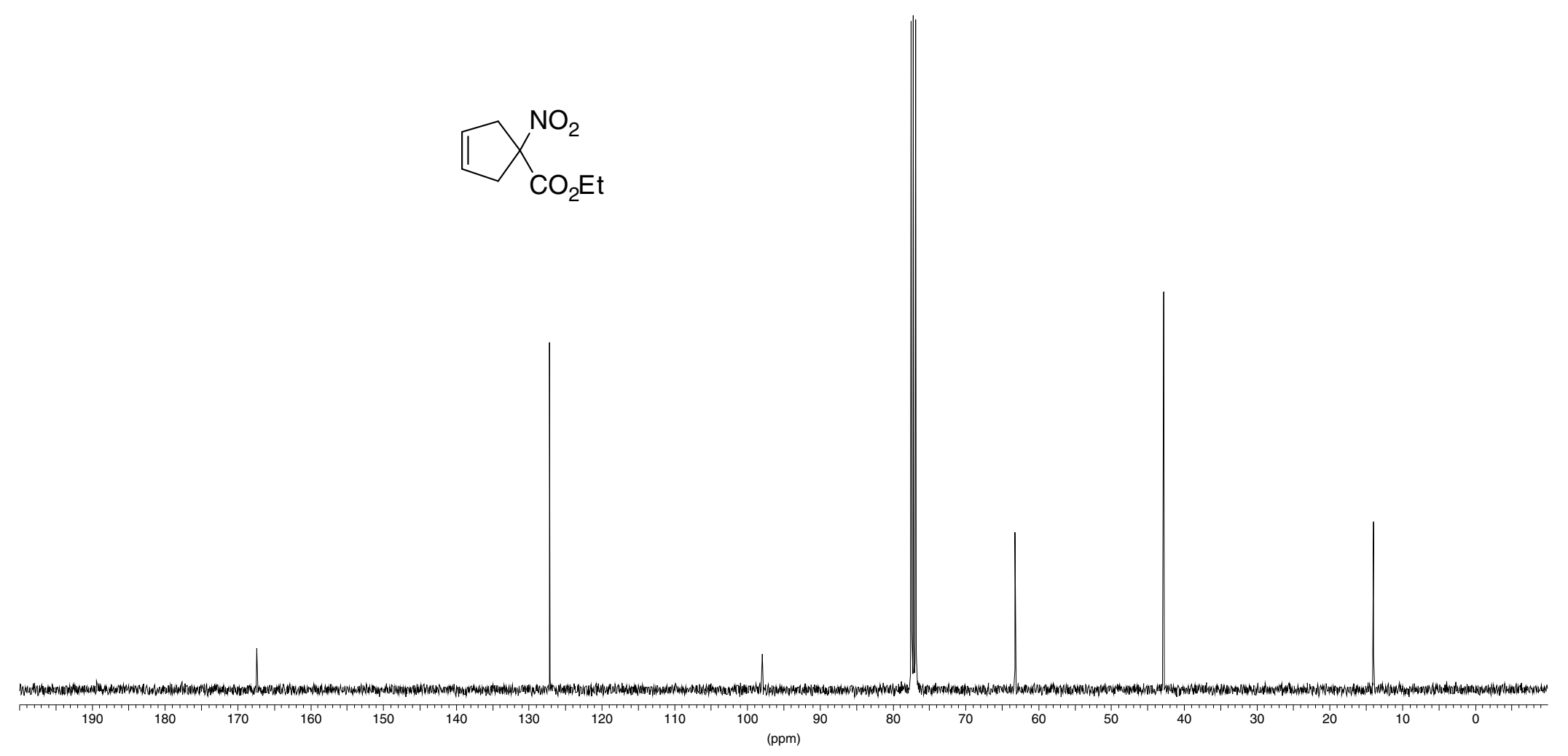


8,8-dimethyl-7,9-dioxaspiro[4.5]dec-2-ene-6,10-dione 16
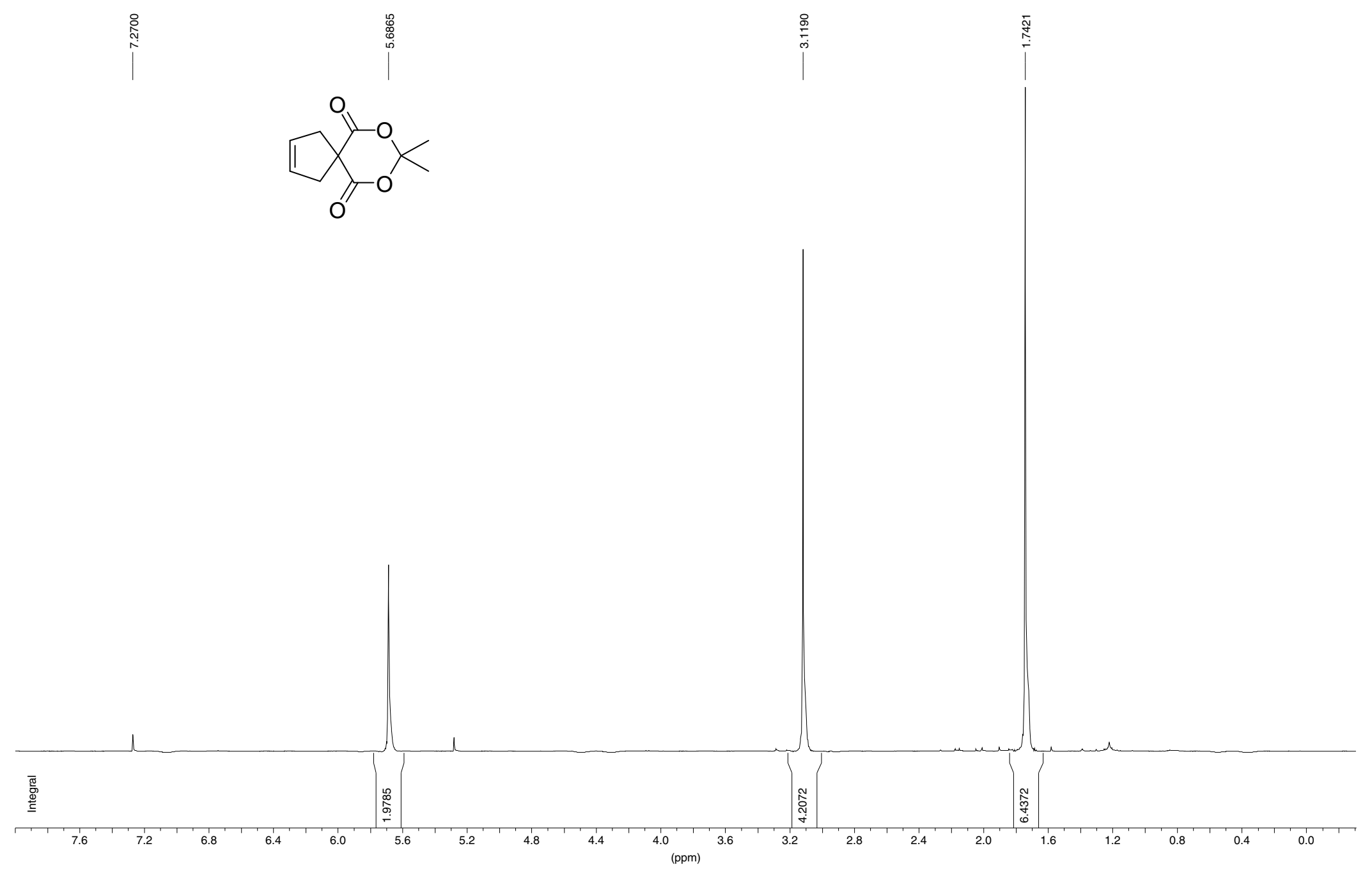
8,8-dimethyl-7,9-dioxaspiro[4.5]dec-2-ene-6,10-dione 16
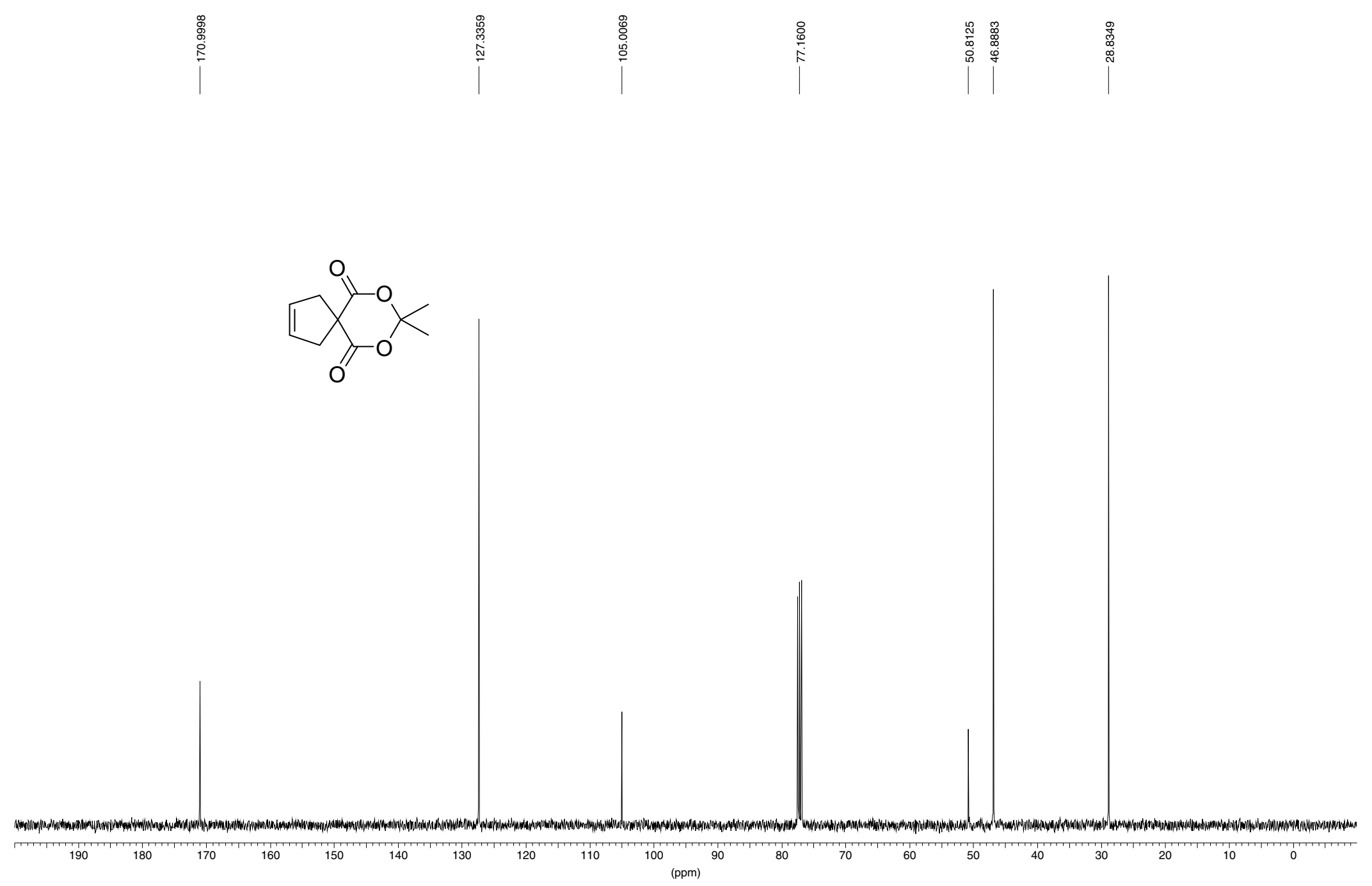
Spiro[4.5]dec-2-ene-6,10-dione 17
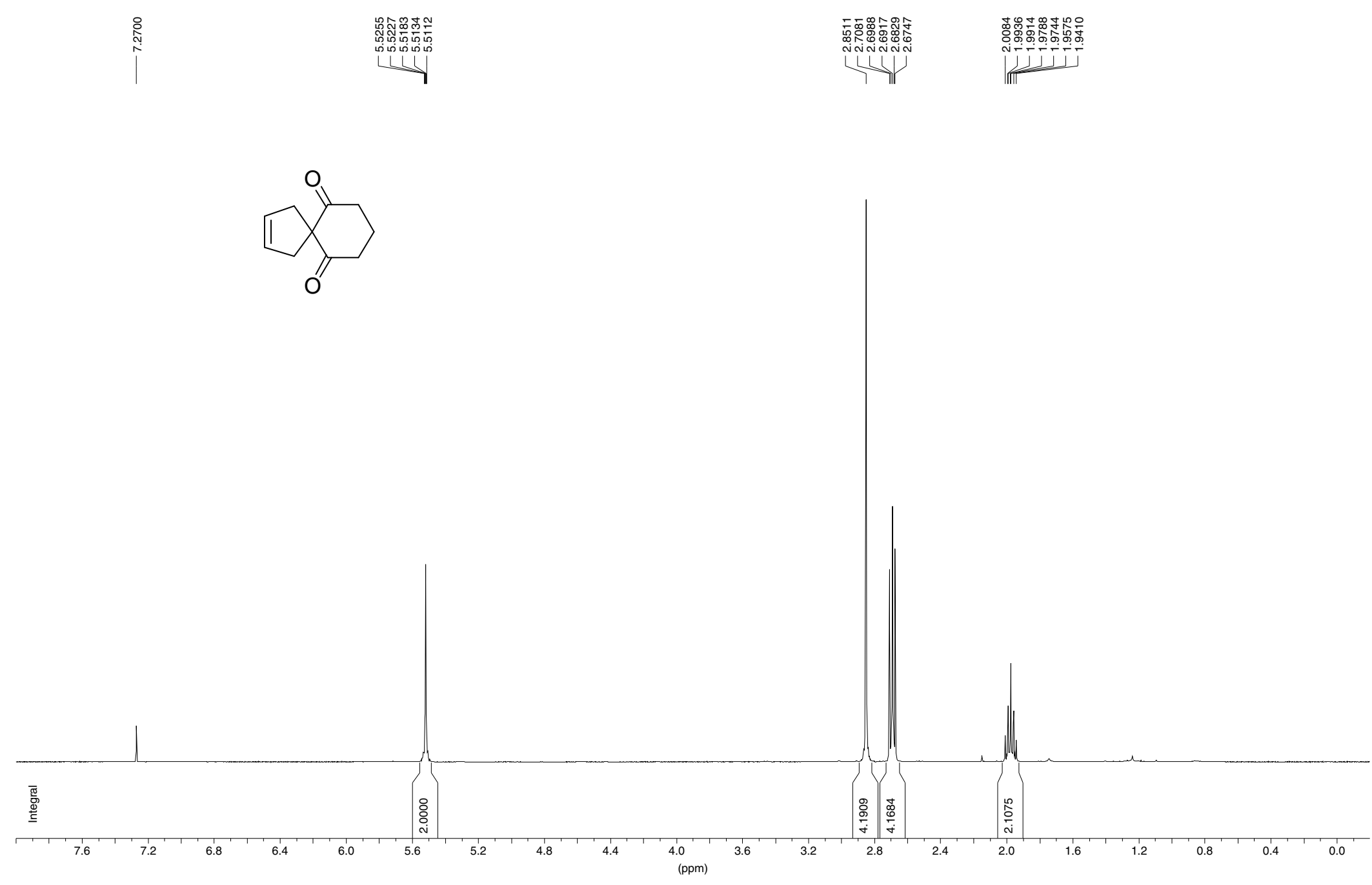
Spiro[4.5]dec-2-ene-6,10-dione 17

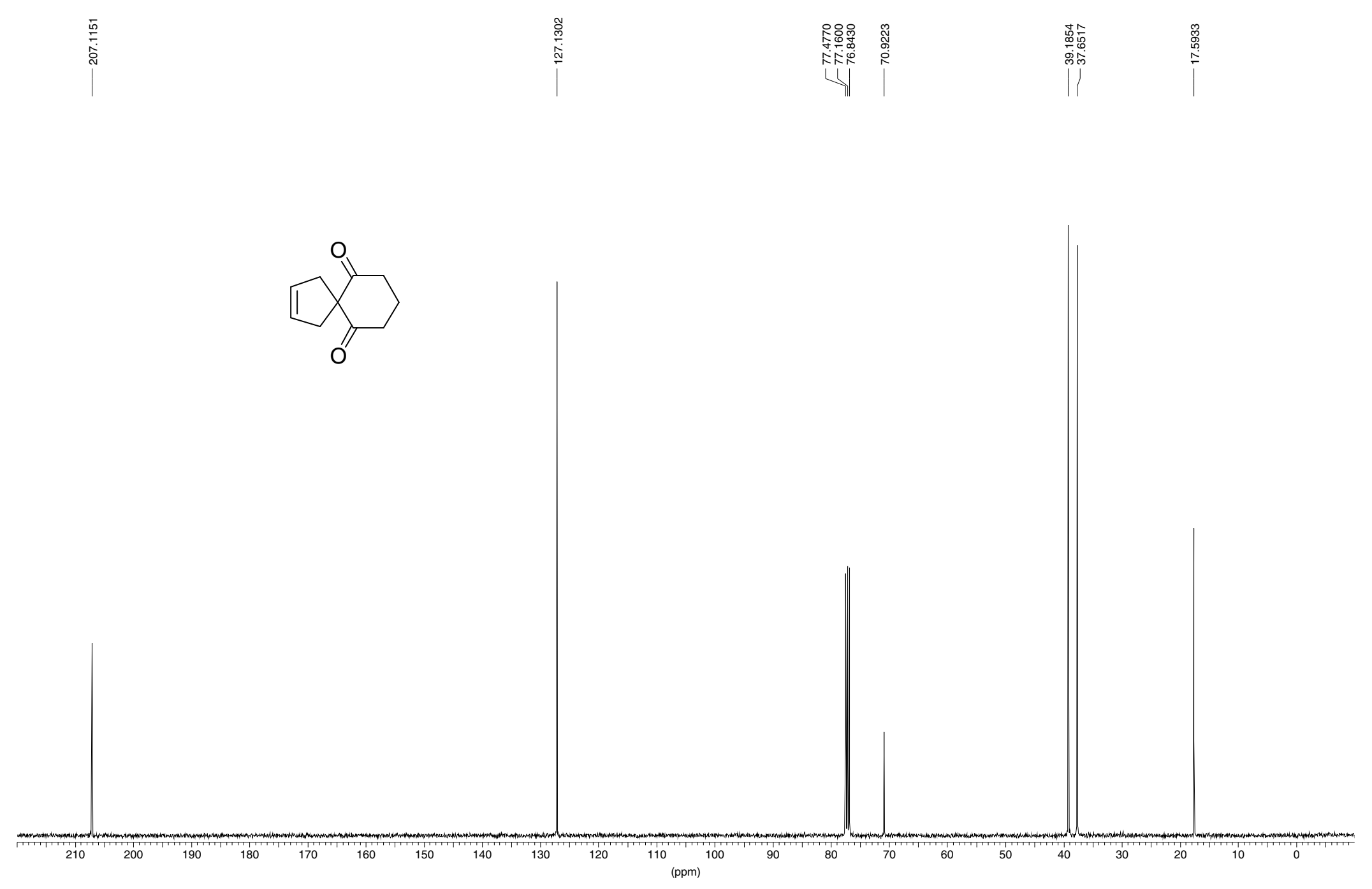


7,9-dimethyl-9-diazaspiro[4.5]dec-2-ene-6,8,10-trione 18
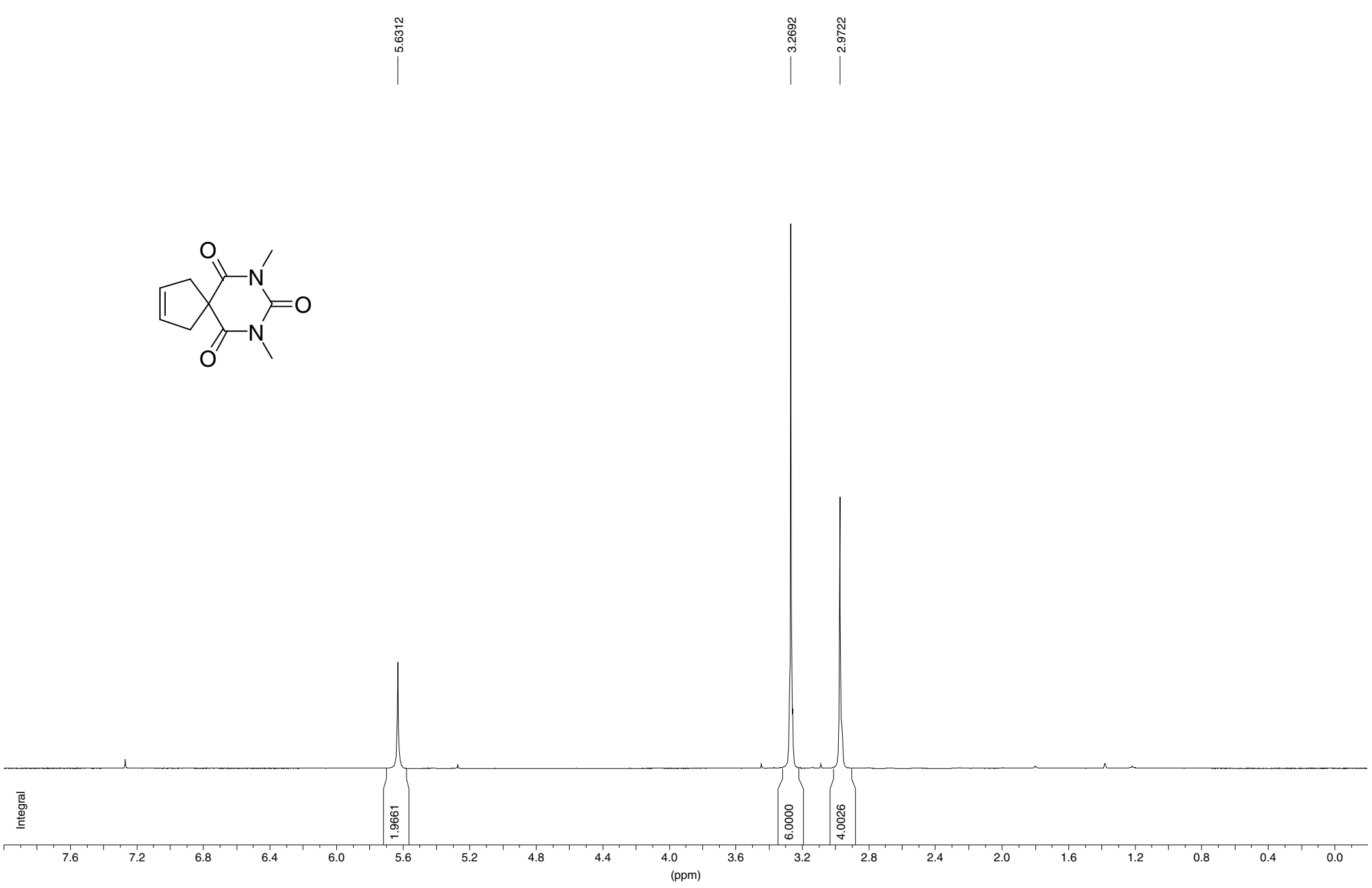
7,9-dimethyl-9-diazaspiro[4.5]dec-2-ene-6,8,10-trione 18
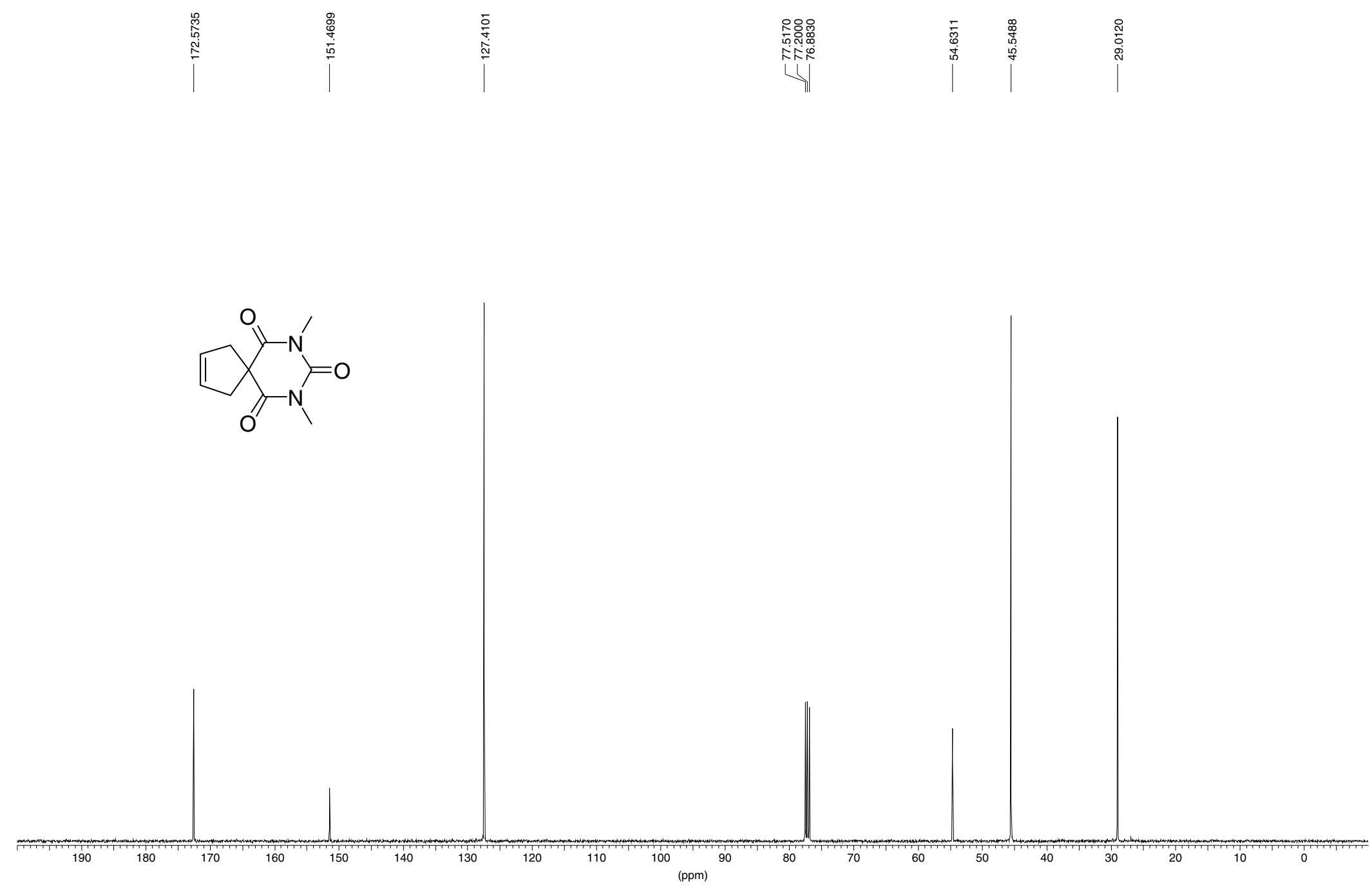